\title{
On the Incommensurability Phenomenon
}

\author{
Donniell E. Fishkind \\ Johns Hopkins University \\ Youngser Park \\ Johns Hopkins University
}

\author{
Cencheng Shen \\ Johns Hopkins University \\ Carey E. Priebe \\ Johns Hopkins University
}

\begin{abstract}
Suppose that two large, multi-dimensional data sets are each noisy measurements of the same underlying random process, and principal components analysis is performed separately on the data sets to reduce their dimensionality. In some circumstances it may happen that the two lower-dimensional data sets have an inordinately large Procrustean fitting-error between them. The purpose of this manuscript is to quantify this "incommensurability phenomenon". In particular, under specified conditions, the square Procrustean fitting-error of the two normalized lowerdimensional data sets is (asymptotically) a convex combination (via a correlation parameter) of the Hausdorff distance between the projection subspaces and the maximum possible value of the square Procrustean fitting-error for normalized data. We show how this gives rise to the incommensurability phenomenon, and we employ illustrative simulations and also use real data to explore how the incommensurability phenomenon may have an appreciable impact.
\end{abstract}

Keywords: Incommensurability phenomenon; Procrustes fitting; Principal components analysis; Grassmannian; Hausdorff distance.

The work of all authors was partially supported by National Security Science and Engineering Faculty Fellowship (NSSEFF), Johns Hopkins University Human Language Technology Center of Excellence (JHU HLT COE), and the XDATA program of the Defense Advanced Research Projects Agency (DARPA) administered through Air Force Research Laboratory contract FA8750-12-2-0303. The authors are grateful to the anonymous referees for particularly useful comments that greatly strengthened this paper.

Corresponding Author's Address: Carey E. Priebe, Department of Applied Mathematics and Statistics, Johns Hopkins University, Baltimore, MD 21218, United States, email: cep@jhu.edu.

Published online: 21 July 2016 


\section{Overview and Outline}

The ever-increasing importance of modern big-data analytics brings with it the imperative to understand fusion and and inference on multiple and massive disparate, distributed data sets. What processing can be profitably done separately, for subsequent joint inference? In the case where each data set consists of measurements on the same objects, and combining the full data sets is prohibitively expensive, it seems reasonable to separately project each large, high-dimensional collection to a low-dimensional space, and to then combine the representations. Unfortunately, this model can lead to undesirable incommensurability with significant deleterious effects on fusion and inference. In this manuscript, we quantify an appearance of this phenomenon.

In Section 2 we begin with an idealized Tale of Two Scientists and its accompanying Theorem 1, in order to pave the way for our main result, Theorem 2-stated and proved in Section 3-wherein, under more general conditions, an asymptotic relationship is given between the Procrustean fitting-error of the two lower dimensional data sets and a distance between the projections.

Then, in Section 4, we perform simulation experiments and utilize real data to illustrate and support our main result Theorem 2, and we use these simulations and real data to explore the implications of Theorem 2; in particular, when two correlated data sets are separately projected to achieve dimension reduction, and when there is an insufficient spectral gap in the covariance structure at the projection-dimension cutoff, then large projection distance may result between the projections for the two data sets, and inordinately large Procrustean fitting-error then follows. This "incommensurability phenomenon" was named in Priebe, Marchette, Ma, and Adali (2013).

\subsection{Background, and an Applied, Takeaway Lesson}

Dimension reduction is often applied to data before subsequent inference. Principal components analysis (PCA) (Anderson 2003; Joliffe 2002) and multi-dimensional scaling (Borg and Groenen 2005) are two traditional tools for data processing; the Big Data trend has motivated many recent advances in dimension reduction, such as nonlinear dimension reduction (Tenenbaum, de Silva, and Langford 2000; Saul and Roweis 2000; Belkin and Niyogi 2003), sparse and robust PCA (Zou and Hastie 2006; Candes, Li, Ma, and Wright 2009; Witten, Tibshirani, and Hastie 2009), etc., which all achieve good performance in their respective domains. For the purpose of this paper, we confine ourselves to principal components analysis, which remains a very popular and successful method for processing data. 
Procrustean fitting-error is a simple-yet useful—statistic for the comparison of two correlated sets of spatial or spatially-embedded data. To give just a few examples, see Sibson (1978) and Sibson (1979) where Procrustes fit is used to assess the goodness-of-fit between two slightly different spatial configurations projected to a lower dimensional space by multi-dimensional scaling. Procrustes analysis is similarly seen to be a valuable tool for manifold alignment in Luo and Hancock (1999), Wang and Mahadevan (2008), and Wang, Liu, Vu, and Mahadevan (2012), and also see Goldberg and Ritov (2009); indeed, Procrustes fit can be used to compare manifold-based embedding algorithms.

Several factors may contribute to the manifestation of the incommensurability phenomenon when two correlated data sets are projected to a lower dimension. One factor is the circumstance where the two data sets are projected separately when the dimension reduction is performed. Another factor is the circumstance where the choice of embedding dimension $d$ does not leave a sufficiently large gap between the $d$ 'th and $d+1$ 'th eigenvalues of the covariance matrix for the data sets. These factors may combine to allow substantial probability of having significant distance between the separate projection subspaces, which then causes an inordinately large Procrustean fitting-error.

Of course, one remedy is simply not to do the projections separately for the two data sets; robust joint embedding schemes are available, such as developed in Wang, Liu, Vu, and Mahadevan (2012), Sharma, Kumar, Daume, and Jacobs (2012), and Priebe, Marchette, Ma, and Adali (2013). Indeed, an easily used candidate is canonical correlation analysis (CCA) (Hotelling 1936; Shen, Sun, Tang, and Priebe 2014), which can be extended to situations where more than two data sets are being treated, and CCA has good properties for subsequent inferential tasks (Sun, Priebe, and Tang 2013; Sun and Priebe 2013; Shen, Sun, Tang, and Priebe 2014). The incommensurability phenomenon can then be avoided at the cost of the extra computation involved, although this extra computation might be a significant burden when dealing with a large volume of data in a distributed system.

Another possible remedy would be to choose the embedding dimension $d$ so as to maintain enough of a gap between the $d$ 'th and $d+1$ 'th eigenvalues of the data sets' covariance matrix. However, this remedy may actually throw out the baby with the bath water; indeed, limiting the embedding dimension to maintain a healthy eigengap may come at the expense of additional signal that might be mined by the inclusion of additional dimensions, if practical.

Besides the theoretical relationships proven in this manuscript, a practical and applied contribution of this manuscript is the takeaway lesson and awareness of the potential danger in not doing joint embedding (or 
similar tactics) in the course of dimension reduction with correlated data sets. Indeed, in the sections that follow, we provide an illustrative vision of what could go wrong.

\section{A Cautionary Tale of Two Scientists}

For this section only, we explore an idealized scenario for the purpose of straightforward illustration; the general setting will be treated in Section 3. For this entire manuscript, a general background reference for matrix analysis tools that we employ (e.g. Procrustes fitting, singular value decomposition, spectral and norm identities and inequalities such as Weyl's Theorem for Hermitian matrices and Interlacing inequalities for Hermitian matrices) is the classical text Horn and Johnson (1990), background on the Grassmannian (e.g. principal angles, Hausdorff distance) useful for our particular work is easily accessible in Qui, Zhang, and Li (2005), and background on principal components analysis (PCA) can be found in Anderson (2003). A classical and broad textbook on the Grassmannian is Chikuse (2003).

Suppose that two scientists each take daily measurements of $m$ features of a random process, where $m$ is a large, positive integer. For each day $i=1,2,3, \ldots$, the first scientist records her daily measurements as $\mathbf{X}^{(i)} \in \mathbb{R}^{m}$, where $\mathbf{X}_{j}^{(i)}$ is her measurement of the $j$ th feature, and the second scientist records his daily measurements as $\mathbf{Y}^{(i)} \in \mathbb{R}^{m}$, where $\mathbf{Y}_{j}^{(i)}$ is his measurement of the $j$ th feature, for $j=1,2, \ldots, m$. Although the two scientists want to record the same process, suppose that their measurements are made with some error, which we model in the following manner.

There are three collections of random variables $\left\{\mathbf{Z}_{j}^{(i)}\right\},\left\{\mathbf{Z}_{j}^{\prime(i)}\right\}$, and $\left\{\mathbf{Z}_{j}^{\prime \prime}(i)\right\}$, each over indices $i=1,2,3, \ldots$ and $j=1,2, \ldots, m$, such that these random variables are all collectively independent and identically distributed, and their common distribution has finite variance $\alpha>0$. Suppose that the random variables $\left\{\mathbf{Z}_{j}^{(i)}\right\}$ are the signal feature values associated with the process that the scientists would like to record, and the random variables $\left\{\mathbf{Z}_{j}^{\prime(i)}\right\}$ and $\left\{\mathbf{Z}_{j}^{\prime \prime(i)}\right\}$ are confounding noise. Let a real-valued "measurementaccuracy" parameter $\gamma$ be fixed in the interval $[0,1]$. One scenario is that for each day $i=1,2,3, \ldots$ and feature $j=1,2, \ldots, m$, the first scientist's measurement $\mathbf{X}_{j}^{(i)}$ is a mixture of $\mathbf{Z}_{j}^{(i)}$ and $\mathbf{Z}_{j}^{\prime(i)}$ with respective probabilities $\gamma$ and $1-\gamma$, and the second scientist's measurement $\mathbf{Y}_{j}^{(i)}$ is a mixture of $\mathbf{Z}_{j}^{(i)}$ and $\mathbf{Z}^{\prime \prime}{ }_{j}^{(i)}$ with respective probabilities $\gamma$ and $1-\gamma$. A second scenario is that, instead, for each day $i=1,2,3, \ldots$ and feature $j=1,2, \ldots, m$, $\mathbf{X}_{j}^{(i)}=\gamma \cdot \mathbf{Z}_{j}^{(i)}+\sqrt{1-\gamma^{2}} \cdot \mathbf{Z}_{j}^{\prime(i)}$ and $\mathbf{Y}_{j}^{(i)}=\gamma \cdot \mathbf{Z}_{j}^{(i)}+\sqrt{1-\gamma^{2}} \cdot \mathbf{Z}^{\prime \prime}{ }_{j}^{(i)}$. The 
main result of Section 2 is Theorem 1, which will hold in either of these two scenarios. At one extreme, if $\gamma=1$, then the two scientists' measurements are perfectly accurate and $\mathbf{X}^{(i)}=\mathbf{Y}^{(i)}$ for all $i$. At the other extreme, if $\gamma=0$, then the two scientists' measurements are independent of each other.

For each positive integer $n$, denote by $X^{(n)}$ the matrix $\left[\mathbf{X}^{(1)}\left|\mathbf{X}^{(2)}\right| \cdots\right.$ $\left.\mathbf{X}^{(n)}\right] \in \mathbb{R}^{m \times n}$ consisting of the first scientist's measurements over the first $n$ days, and denote by $Y^{(n)}$ the matrix $\left[\mathbf{Y}^{(1)}\left|\mathbf{Y}^{(2)}\right| \cdots \mathbf{Y}^{(n)}\right] \in \mathbb{R}^{m \times n}$ consisting of the second scientist's measurements over the first $n$ days.

Because the measurement vectors are in high-dimensional space $\mathbb{R}^{m}$, suppose the scientists project their respective measurement vectors to the lower-dimensional space $\mathbb{R}^{k}$ for some smaller, positive integer $k$. This is done in the following manner. Let $H_{n}=I_{n}-\frac{1}{n} J_{n}$ denote the centering matrix ( $I_{n}$ and $J_{n}$ are, respectively, the $n \times n$ identity matrix and the matrix of all ones). Suppose that the first scientist chooses a sequence $\mathcal{A}^{(1)}, \mathcal{A}^{(2)}, \mathcal{A}^{(3)}, \ldots$ of random (or deterministic) elements of the Grassmannian $\mathcal{G}_{k, m}$ (the space of all $k$-dimensional subspaces of $\mathbb{R}^{m}$ ), and suppose that the second scientist chooses a sequence $\mathcal{B}^{(1)}, \mathcal{B}^{(2)}, \mathcal{B}^{(3)}, \ldots$ of random (or deterministic) elements of the Grassmannian $\mathcal{G}_{k, m}$. No assumptions are made on the distributions of these elements of the Grassmannian or on their dependence/independence, but one example of interest is where, for $n=1,2,3, \ldots, \mathcal{A}^{(n)}, \mathcal{B}^{(n)} \in \mathcal{G}_{k, m}$ denote the respective $k$-dimensional subspaces to which principal components analysis (PCA) projects $X^{(n)} H_{n}$ and $Y^{(n)} H_{n}$, respectively (and separately). Let $P_{\mathcal{A}^{(n)}}$ denote the projection operator from $\mathbb{R}^{m}$ onto $\mathcal{A}^{(n)}$. On each day $n$, the first scientist reports the scaled matrix $\mathcal{X}^{(n)}:=\frac{\sqrt{k}}{\left\|P_{\mathcal{A}^{(n)}} X^{(n)} H_{n}\right\|_{F}} P_{\mathcal{A}^{(n)}} X^{(n)} H_{n} \in \mathbb{R}^{k \times n}$ to the Governing Board of Scientists, and the second scientist reports the scaled matrix $\mathcal{Y}^{(n)}:=\frac{\sqrt{k}}{\left\|P_{\mathcal{B}^{(n)}} Y^{(n)} H_{n}\right\|_{F}} P_{\mathcal{B}^{(n)}} Y^{(n)} H_{n} \in \mathbb{R}^{k \times n}$ to the Governing Board of Scientists, where $\|\cdot\|_{F}$ denotes the Froebenius norm. (Nota Bene: The specific choice of $\sqrt{k}$ in the scaling $\left\|\mathcal{X}^{(n)}\right\|_{F}=\left\|\mathcal{Y}^{(n)}\right\|_{F}=\sqrt{k}$ is an innocuous notational convenience.)

Now, the Governing Board of Scientists wants to perform its own check that the two scientists are indeed taking measurements reflecting the same process. So the Governing Board of Scientists computes the Procrustean fitting-error $\epsilon\left(\mathcal{X}^{(n)}, \mathcal{Y}^{(n)}\right):=\min _{Q \in \mathbb{R}^{k \times k}: Q^{T} Q=I_{k}}\left\|Q \mathcal{X}^{(n)}-\mathcal{Y}^{(n)}\right\|_{F}$. It will later be seen (from Equation (5)) that the square Procrustean fittingerror satisfies $0 \leq \epsilon^{2}\left(\mathcal{X}^{(n)}, \mathcal{Y}^{(n)}\right) \leq 2 k$; the Governing Board of Scientists reasons that this square Procrustean fitting-error should be small (negligible compared to $2 k$ ) if indeed $\gamma$ is close to 1 . Is this reasoning valid?

In the following, $d(\cdot, \cdot)$ denotes the Hausdorff distance (e.g. see Qiu, Zhang, and Li 2005) on the Grassmannian $\mathcal{G}_{k, m}$; in particular, for any $\mathcal{A}, \mathcal{B} \in$ $\mathcal{G}_{k, m}, d(\mathcal{A}, \mathcal{B})=\sqrt{\sum_{i=1}^{k}\left(2 \sin \frac{\theta_{i}(\mathcal{A}, \mathcal{B})}{2}\right)^{2}}$ where $\left\{\theta_{i}(\mathcal{A}, \mathcal{B})\right\}_{i=1}^{k}$ are the prin- 
cipal angles between $\mathcal{A}$ and $\mathcal{B}$. Note that the square Hausdorff distance satisfies $0 \leq d^{2}(\mathcal{A}, \mathcal{B}) \leq 2 k$.

Theorem 1. Almost surely, $\epsilon^{2}\left(\mathcal{X}^{(n)}, \mathcal{Y}^{(n)}\right)-\left[\left(1-\gamma^{2}\right) \cdot 2 k+\gamma^{2} \cdot d^{2}\left(\mathcal{A}^{(n)}, \mathcal{B}^{(n)}\right)\right]$ $\rightarrow 0$ as $n \rightarrow \infty$.

The proof of Theorem 1 is given later, in Section 3.2, as a special case of the more general Theorem 2 .

Theorem 1 says that $\epsilon^{2}\left(\mathcal{X}^{(n)}, \mathcal{Y}^{(n)}\right)$ asymptotically becomes this convex combination (via $\gamma^{2}$ ) of $2 k$ and $d^{2}\left(\mathcal{A}^{(n)}, \mathcal{B}^{(n)}\right)$. In particular, if $\gamma$ is close to 0 , which implies that the two scientists' measurements are independent of each other, then indeed $\epsilon^{2}\left(\mathcal{X}^{(n)}, \mathcal{Y}^{(n)}\right)$ is close to its maximum possible value $2 k$, but if $\gamma$ is close to 1 , meaning that the scientists' measurements are close to being the same as each other, we then have $\epsilon^{2}\left(\mathcal{X}^{(n)}, \mathcal{Y}^{(n)}\right)$ close to $d^{2}\left(\mathcal{A}^{(n)}, \mathcal{B}^{(n)}\right)$. Is this square Hausdorff distance close to zero when $\gamma$ is close to 1 ?

In Section 4 we show that, in fact, if the (separate) principal components analysis projections are used then this may not be the case, and the square Hausdorff distance $d^{2}\left(\mathcal{A}^{(n)}, \mathcal{B}^{(n)}\right)$ might even be close to its maximum possible value of $2 k$. By contrast, here if the two scientists both used the simple-minded projection consisting of just taking the first $k$ coordinates of $\mathbb{R}^{m}$ and ignoring the rest of the coordinates, then $d^{2}\left(\mathcal{A}^{(n)}, \mathcal{B}^{(n)}\right)=0$, in which case $\gamma$ close to 1 would indeed yield $\epsilon^{2}\left(\mathcal{X}^{(n)}, \mathcal{Y}^{(n)}\right)$ close to 0 .

\section{The Asymptotic Relationship Between Procrustean Fitting-Error and the Projection Distance}

The main result of this section is the statement and proof of Theorem 2. We begin with a description of a general setting and a list of basic facts that will be used in the proof of Theorem 2 .

\subsection{Preliminaries and the General Setting}

From this point and on, we will consider a much more general setting than the idealistic setting of Section 2. Suppose now that $\mathbf{X}^{(1)}, \mathbf{X}^{(2)}, \mathbf{X}^{(3)}$, $\ldots \in \mathbb{R}^{m}$ and $\mathbf{Y}^{(1)}, \mathbf{Y}^{(2)}, \mathbf{Y}^{(3)}, \ldots \in \mathbb{R}^{m}$ are random vectors (for convenience, let us denote $\mathbf{X} \equiv \mathbf{X}^{(1)}, \mathbf{Y} \equiv \mathbf{Y}^{(1)}$ ) such that the stacked random vectors $\left[\begin{array}{c}\mathbf{X}^{(1)} \\ \mathbf{Y}^{(1)}\end{array}\right],\left[\begin{array}{l}\mathbf{X}^{(2)} \\ \mathbf{Y}^{(2)}\end{array}\right],\left[\begin{array}{l}\mathbf{X}^{(3)} \\ \mathbf{Y}^{(3)}\end{array}\right], \ldots \in \mathbb{R}^{2 m}$ are independent, identically distributed, with covariance matrix

$$
\operatorname{Cov}\left[\begin{array}{l}
\mathbf{X} \\
\mathbf{Y}
\end{array}\right]=\left[\begin{array}{cc}
\operatorname{Cov}(\mathbf{X}) & \operatorname{Cov}(\mathbf{X}, \mathbf{Y}) \\
\operatorname{Cov}(\mathbf{Y}, \mathbf{X}) & \operatorname{Cov}(\mathbf{Y})
\end{array}\right] \in \mathbb{R}^{2 m \times 2 m}
$$

(We no longer require, in the manner of Section 2, that $\mathbf{X}$ and $\mathbf{Y}$ have independent, nor identically distributed components, nor that they arise as a mix- 
ture of other random variables in any particular way.) Assume that $\operatorname{Cov}(\mathbf{X})$ and $\operatorname{Cov}(\mathbf{Y})$ are both nonzero matrices.

Then define, for each positive integer $n$, random matrix $X^{(n)}:=$ $\left[\mathbf{X}^{(1)}\left|\mathbf{X}^{(2)}\right| \cdots \mathbf{X}^{(n)}\right] \in \mathbb{R}^{m \times n}$ and $Y^{(n)}:=\left[\mathbf{Y}^{(1)}\left|\mathbf{Y}^{(2)}\right| \cdots \mathbf{Y}^{(n)}\right] \in \mathbb{R}^{m \times n}$. Let $\mathcal{A}^{(n)} \in \mathcal{G}_{k, m}$ denote the $k$-dimensional subspace to which principal components analysis (PCA) projects $X^{(n)} H_{n}$, and let $\mathcal{B}^{(n)} \in \mathcal{G}_{k, m}$ denote the $k$-dimensional subspace to which principal components analysis (PCA) projects $Y^{(n)} H_{n}$ (these projections being done separately). In the special case where $\operatorname{Cov}(\mathbf{X})$ and $\operatorname{Cov}(\mathbf{Y})$ are scalar multiples of $I_{m}$, then we will explicitly allow $\left\{\mathcal{A}^{(n)}\right\}_{n=1}^{\infty},\left\{\mathcal{B}^{(n)}\right\}_{n=1}^{\infty}$ to be any sequences of elements in $\mathcal{G}_{k, m}$ whatsoever, deterministic or random.

It is useful to consider the projections $P_{\mathcal{A}^{(n)}}$ and $P_{\mathcal{B}^{(n)}}$ as $m \times m$ symmetric, idempotent matrices (i.e., keep the ambient coordinate system $\mathbb{R}^{m}$ for the projection's range) and, for each $n=1,2, \ldots$, define

$$
\begin{aligned}
\mathcal{X}^{(n)} & =\frac{\sqrt{k}}{\left\|P_{\mathcal{A}^{(n)}} X^{(n)} H_{n}\right\|_{F}} P_{\mathcal{A}^{(n)}} X^{(n)} H_{n} \in \mathbb{R}^{m \times n} \\
\mathcal{Y}^{(n)} & :=\frac{\sqrt{k}}{\left\|P_{\mathcal{B}^{(n)}} Y^{(n)} H_{n}\right\|_{F}} P_{\mathcal{B}^{(n)}} Y^{(n)} H_{n} \in \mathbb{R}^{m \times n} .
\end{aligned}
$$

(There is no difference for our results and for the Procrustean fitting-error if, as in Section 2, we instead treated $P_{\mathcal{A}^{(n)}}$ and $P_{\mathcal{B}^{(n)}}$ as functions $\mathbb{R}^{m} \rightarrow \mathbb{R}^{k}$ with the coordinate systems of $\mathcal{A}^{(n)}$ and $\mathcal{B}^{(n)}$, respectively, in which case we have $\mathcal{X}^{(n)}$ and $\mathcal{Y}^{(n)}$ in $\mathbb{R}^{k \times n}$ instead of $\mathbb{R}^{m \times n}$.)

For any matrix $C \in \mathbb{R}^{m \times m}$ with only real-valued eigenvalues (e.g., symmetric matrices), let $\lambda_{1}(C) \geq \lambda_{2}(C) \geq \cdots \geq \lambda_{m}(C)$ denote the eigenvalues of $C$. For any matrix $C \in \mathbb{R}^{m \times n}$, let $\sigma_{1}(C) \geq \sigma_{2}(C) \geq \cdots \geq$ $\sigma_{\min \{m, n\}}(C)$ denote the singular values of $C$. Recall that if $C$ is symmetric and positive semidefinite (e.g., a covariance matrix) then $\lambda_{i}(C)=$ $\sigma_{i}(C)$ for all $i=1,2, \ldots, m$, and recall that, for any $C \in \mathbb{R}^{m \times n}, D \in$ $\mathbb{R}^{n \times m}$, the nonzero eigenvalues of $C D$ are the same as the nonzero eigenvalues of $D C$. For any $\mathcal{A}, \mathcal{B} \in \mathcal{G}_{k, m}$ (with projection matrices $P_{\mathcal{A}}, P_{\mathcal{B}}$ ) and all $i=1,2, \ldots, m$, we thus have $\sigma_{i}^{2}\left(P_{\mathcal{A}} P_{\mathcal{B}}\right)=\lambda_{i}\left(P_{\mathcal{A}} P_{\mathcal{B}} P_{\mathcal{B}}^{T} P_{\mathcal{A}}^{T}\right)=$ $\lambda_{i}\left(P_{\mathcal{A}} P_{\mathcal{B}} P_{\mathcal{B}} P_{\mathcal{A}}\right)=\lambda_{i}\left(P_{\mathcal{A}} P_{\mathcal{A}} P_{\mathcal{B}} P_{\mathcal{B}}\right)=\lambda_{i}\left(P_{\mathcal{A}} P_{\mathcal{B}}\right)$. In fact, $P_{\mathcal{A}} P_{\mathcal{B}}$ has at most $k$ positive eigenvalues and at most $k$ positive singular values (the rest of the eigenvalues and the rest of the singular values are all zero) and, for all $i=1,2, \ldots, k$,

$$
\sigma_{i}\left(P_{\mathcal{A}} P_{\mathcal{B}}\right)=\sqrt{\lambda_{i}\left(P_{\mathcal{A}} P_{\mathcal{B}}\right)}=\cos \theta_{i}(\mathcal{A}, \mathcal{B})
$$

where $\left\{\theta_{i}(\mathcal{A}, \mathcal{B})\right\}_{i=1}^{k}$ are the principal angles between $\mathcal{A}$ and $\mathcal{B}$.

For each $n=1,2, \ldots$, the Hausdorff distance $d\left(\mathcal{A}^{(n)} \mathcal{B}^{(n)}\right)$ is the nonnegative square root of 


$$
\begin{aligned}
d^{2}\left(\mathcal{A}^{(n)}, \mathcal{B}^{(n)}\right) & :=\sum_{i=1}^{k} 2^{2} \sin ^{2}\left(\frac{\theta_{i}\left(\mathcal{A}^{(n)}, \mathcal{B}^{(n)}\right)}{2}\right) \\
& =\sum_{i=1}^{k} 2\left(1-\cos \theta_{i}\left(\mathcal{A}^{(n)}, \mathcal{B}^{(n)}\right)\right) .
\end{aligned}
$$

It is clear that $0 \leq d^{2}\left(\mathcal{A}^{(n)}, \mathcal{B}^{(n)}\right) \leq 2 k$. We also define, for each $n=$ $1,2, \ldots$, the quantity

$$
\begin{aligned}
& \check{\partial}^{2}\left(\mathcal{A}^{(n)}, \mathcal{B}^{(n)}\right):= \\
& \quad \sum_{i=1}^{k} 2\left(1-\frac{1}{\frac{1}{k} \sum_{j=1}^{k} \sigma_{j}(\operatorname{Cov}(\mathbf{X}, \mathbf{Y}))} \sigma_{i}\left(P_{\mathcal{A}^{(n)}} \operatorname{Cov}(\mathbf{X}, \mathbf{Y}) P_{\mathcal{B}^{(n)}}\right)\right)(3)
\end{aligned}
$$

Later, in Proposition 6, we will prove it always holds that $0 \leq \partial^{2}\left(\mathcal{A}^{(n)}, \mathcal{B}^{(n)}\right)$ $\leq 2 k$. Note that if $\operatorname{Cov}(\mathbf{X}, \mathbf{Y})$ is a nonzero scalar multiple of $I_{m}$ then $\widetilde{\partial}^{2}\left(\mathcal{A}^{(n)}, \mathcal{B}^{(n)}\right)$ is equal to $d^{2}\left(\mathcal{A}^{(n)}, \mathcal{B}^{(n)}\right)$ and, in fact, if $\operatorname{Cov}(\mathbf{X}, \mathbf{Y})$ is the zero matrix then we will define $\partial^{2}\left(\mathcal{A}^{(n)}, \mathcal{B}^{(n)}\right) \equiv d^{2}\left(\mathcal{A}^{(n)}, \mathcal{B}^{(n)}\right)$ (because, indeed, $\frac{1}{\frac{1}{k} \sum_{j=1}^{k} \sigma_{j}(\operatorname{Cov}(\mathbf{X}, \mathbf{Y}))}$ is not defined). For this reason, we like to view $\partial^{2}\left(\mathcal{A}^{(n)}, \mathcal{B}^{(n)}\right)$ as a weighted form of the square Hausdorff distance.

For each $n=1,2, \ldots$, the Procrustean fitting-error is defined to be

$$
\epsilon\left(\mathcal{X}^{(n)}, \mathcal{Y}^{(n)}\right):=\min _{Q \in \mathbb{R}^{m \times m}: Q^{T} Q=I_{m}}\left\|Q \mathcal{X}^{(n)}-\mathcal{Y}^{(n)}\right\|_{F} .
$$

In fact, it holds that

$$
\begin{aligned}
\epsilon^{2}\left(\mathcal{X}^{(n)}, \mathcal{Y}^{(n)}\right) & =\left\|\mathcal{X}^{(n)}\right\|_{F}^{2}+\left\|\mathcal{Y}^{(n)}\right\|_{F}^{2}-2 \sum_{i=1}^{m} \sigma_{i}\left(\mathcal{Y}^{(n)} \mathcal{X}^{(n) T}\right) \\
& =2 k-2 \sum_{i=1}^{m} \sigma_{i}\left(\mathcal{Y}^{(n)} \mathcal{X}^{(n) T}\right) .
\end{aligned}
$$

\subsection{The Result}

Within the setting of Section 3.1, we now state and prove the main result of Section 3:

Theorem 2. In the setting of Section 3.1, it holds almost surely that

$$
\epsilon^{2}\left(\mathcal{X}^{(n)}, \mathcal{Y}^{(n)}\right)-\left[(1-\rho) \cdot 2 k+\rho \cdot \partial^{2}\left(\mathcal{A}^{(n)}, \mathcal{B}^{(n)}\right)\right] \rightarrow 0
$$

as $n \rightarrow \infty$, where $\rho$ is defined as

$$
\rho:=\frac{\sum_{j=1}^{k} \sigma_{j}(\operatorname{Cov}(\mathbf{X}, \mathbf{Y}))}{\sqrt{\sum_{j=1}^{k} \sigma_{j}(\operatorname{Cov}(\mathbf{X}))} \sqrt{\sum_{j=1}^{k} \sigma_{j}(\operatorname{Cov}(\mathbf{Y}))}} .
$$


In Proposition 7 we prove that $0 \leq \rho \leq 1$. To prove Theorem 2 we first establish Lemmas 3 and 4:

Lemma 3. Almost surely,

$\operatorname{trace} \frac{1}{n-1} P_{\mathcal{A}^{(n)}} X^{(n)} H_{n} H_{n}^{T} X^{(n) T} P_{\mathcal{A}^{(n)}}^{T} \rightarrow \sum_{i=1}^{k} \sigma_{i}(\operatorname{Cov}(\mathbf{X}))$ as $n \rightarrow \infty$.

Proof of Lemma 3: For each $n=1,2,3, \ldots$, let us consider a singular value decomposition

$$
X^{(n)} H_{n}=U^{(n)} \Lambda^{(n)} V^{(n) T}
$$

where $U^{(n)} \in \mathbb{R}^{m \times m}$ is orthogonal, $\Lambda^{(n)} \in \mathbb{R}^{m \times n}$ is a "diagonal" matrix, with nonnegative diagonals non-increasing along its main diagonal, and $V^{(n)} \in \mathbb{R}^{n \times n}$ is orthogonal. By the definition of PCA,

$$
P_{\mathcal{A}^{(n)}} X^{(n)} H_{n}=U^{(n)} E \Lambda^{(n)} V^{(n) T},
$$

where $E \in \mathbb{R}^{m \times m}$ is the diagonal matrix with its first $k$ diagonals 1 and its remaining diagonals 0 . Thus, the matrix

$$
X^{(n)} H_{n} H_{n}^{T} X^{(n)^{T}}=U^{(n)} \Lambda^{(n)} \Lambda^{(n) T} U^{(n) T}
$$

and the matrix

$$
P_{\mathcal{A}^{(n)}} X^{(n)} H_{n} H_{n}^{T} X^{(n)^{T}} P_{\mathcal{A}^{(n)}}^{T}=U^{(n)} E \Lambda^{(n)} \Lambda^{(n) T} E U^{(n) T}
$$

share their $k$ largest eigenvalues, and the remaining $m-k$ eigenvalues of the latter matrix are 0. By the Strong Law of Large Numbers, almost surely $\frac{1}{n-1} X^{(n)} H_{n} H_{n}^{T} X^{(n)^{T}} \rightarrow \operatorname{Cov}(\mathbf{X})$, hence we have

$$
\begin{gathered}
\operatorname{trace} \frac{1}{n-1} P_{\mathcal{A}^{(n)}} X^{(n)} H_{n} H_{n}^{T} X^{(n) T} P_{\mathcal{A}^{(n)}}^{T} \rightarrow \sum_{i=1}^{k} \lambda_{i}(\operatorname{Cov}(\mathbf{X})) \\
=\sum_{i=1}^{k} \sigma_{i}(\operatorname{Cov}(\mathbf{X})) \text { as } n \rightarrow \infty
\end{gathered}
$$

Lastly, recall that we explicitly allow $\left\{\mathcal{A}^{(n)}\right\}_{n=1}^{\infty}$ to be any elements of $\mathcal{G}_{k, m}$ in the special case that $\operatorname{Cov}(\mathbf{X})=\alpha \cdot I_{m}$ for some $\alpha>0$; indeed, in this special case, note that by the boundedness of $\left\{P_{\mathcal{A}^{(n)}}\right\}_{n=1}^{\infty}$ and the Strong Law of Large Numbers that, as $n \rightarrow \infty$,

$$
\begin{aligned}
& \operatorname{trace} \frac{1}{n-1} P_{\mathcal{A}^{(n)}} X^{(n)} H_{n} H_{n}^{T} X^{(n) T} P_{\mathcal{A}^{(n)}}^{T} \\
& =\alpha \cdot \operatorname{trace} P_{\mathcal{A}^{(n)}}+\operatorname{trace} P_{\mathcal{A}^{(n)}}\left(\frac{1}{n-1} X^{(n)} H_{n} H_{n}^{T} X^{(n)^{T}}-\alpha \cdot I_{m}\right) P_{\mathcal{A}^{(n)}}^{T} \\
& \quad \rightarrow \alpha k=\sum_{i=1}^{k} \sigma_{i}(\operatorname{Cov}(\mathbf{X}))
\end{aligned}
$$

as desired. 
Lemma 4. For $i=1,2, \ldots, m$, almost surely

$$
\sigma_{i}^{2}\left(\mathcal{Y}^{(n)} \mathcal{X}^{(n) T}\right)-\delta \cdot \sigma_{i}^{2}\left(P_{\mathcal{A}^{(n)}} \operatorname{Cov}(\mathbf{X}, \mathbf{Y}) P_{\mathcal{B}^{(n)}}\right) \rightarrow 0
$$

as $n \rightarrow \infty$, where $\delta:=\frac{1}{\frac{1}{k} \sum_{j=1}^{k} \sigma_{j}(\operatorname{Cov}(\mathbf{X})) \cdot \frac{1}{k} \sum_{j=1}^{k} \sigma_{j}(\operatorname{Cov}(\mathbf{Y}))}$

Proof of Lemma 4: For each $n=1,2, \ldots$, expand the expression $\mathcal{Y}^{(n)} \mathcal{X}^{(n) T}$ $\left(\mathcal{Y}^{(n)} \mathcal{X}^{(n) T}\right)^{T}$ by the definitions to write it as $\mathcal{Y}^{(n)} \mathcal{X}^{(n) T}\left(\mathcal{Y}^{(n)} \mathcal{X}^{(n) T}\right)^{T}=$ $\phi^{(n)} \cdot \Phi^{(n)}$ where $\phi^{(n)}$ and $\Phi^{(n)}$ are defined by

$\phi^{(n)}:=$

$\frac{k^{2}}{\operatorname{trace} \frac{1}{n-1} P_{\mathcal{B}^{(n)}} Y^{(n)} H_{n} H_{n}^{T} Y^{(n) T} P_{\mathcal{B}^{(n)}}^{T} \cdot \operatorname{trace} \frac{1}{n-1} P_{\mathcal{A}^{(n)}} X^{(n)} H_{n} H_{n}^{T} X^{(n) T} P_{\mathcal{A}^{(n)}}^{T}} \in \mathbb{R}$

and

$$
\begin{aligned}
& \Phi^{(n)}:= \\
& P_{\mathcal{B}^{(n)}}\left(\frac{1}{n-1} Y^{(n)} H_{n} H_{n}^{T} X^{(n) T}\right) P_{\mathcal{A}^{(n)}}^{T} P_{\mathcal{A}^{(n)}}\left(\frac{1}{n-1} X^{(n)} H_{n} H_{n}^{T} Y^{(n) T}\right) P_{\mathcal{B}^{(n)}}^{T} \\
& \quad \in \mathbb{R}^{m \times m} .
\end{aligned}
$$

Define $\Psi_{X, Y}^{(n)}:=\frac{1}{n-1} X^{(n)} H_{n} H_{n}^{T} Y^{(n) T}-\operatorname{Cov}(\mathbf{X}, \mathbf{Y})$; by the Strong Law of Large Numbers, almost surely $\Psi_{X, Y}^{(n)} \rightarrow 0$ as $n \rightarrow \infty$. Thus, by the subadditivity and submultiplicativity of the norm, and by the boundedness of $\left\{P_{\mathcal{A}^{(n)}}\right\}_{n=1}^{\infty}$ and $\left\{P_{\mathcal{B}^{(n)}}\right\}_{n=1}^{\infty}$, we have almost surely that

$$
\begin{aligned}
\| \Phi^{(n)}- & P_{\mathcal{B}^{(n)}} \operatorname{Cov}^{T}(\mathbf{X}, \mathbf{Y}) P_{\mathcal{A}^{(n)}}^{T} P_{\mathcal{A}^{(n)}} \operatorname{Cov}(\mathbf{X}, \mathbf{Y}) P_{\mathcal{B}^{(n)}}^{T} \|_{F} \\
& =\| P_{\mathcal{B}^{(n)}} \Psi_{X, Y}^{(n) T} P_{\mathcal{A}^{(n)}}^{T} P_{\mathcal{A}^{(n)}} \Psi_{X, Y}^{(n)} P_{\mathcal{B}^{(n)}}^{T} \\
& +P_{\mathcal{B}^{(n)}} \Psi_{X, Y}^{(n) T} P_{\mathcal{A}^{(n)}}^{T} P_{\mathcal{A}^{(n)}} \operatorname{Cov}(\mathbf{X}, \mathbf{Y}) P_{\mathcal{B}^{(n)}}^{T} \\
& +P_{\mathcal{B}^{(n)}} \operatorname{Cov}^{T}(\mathbf{X}, \mathbf{Y}) P_{\mathcal{A}^{(n)}}^{T} P_{\mathcal{A}^{(n)}} \Psi_{X, Y}^{(n)} P_{\mathcal{B}^{(n)}}^{T} \|_{F} \rightarrow 0
\end{aligned}
$$

as $n \rightarrow \infty$. Now, by Lemma 3 and the definition of $\phi^{(n)}$, almost surely $\phi^{(n)} \rightarrow \delta$ as $n \rightarrow \infty$, hence by (6) and the boundedness of $\left\{P_{\mathcal{A}^{(n)}}\right\}_{n=1}^{\infty}$ and $\left\{P_{\mathcal{B}^{(n)}}\right\}_{n=1}^{\infty}$, we have almost surely that

$$
\begin{aligned}
& \left\|\phi^{(n)} \cdot \Phi^{(n)}-\delta \cdot P_{\mathcal{B}^{(n)}} \operatorname{Cov}^{T}(\mathbf{X}, \mathbf{Y}) P_{\mathcal{A}^{(n)}}^{T} P_{\mathcal{A}^{(n)}} \operatorname{Cov}(\mathbf{X}, \mathbf{Y}) P_{\mathcal{B}^{(n)}}^{T}\right\|_{F} \\
& \quad \leq\left\|\phi^{(n)}\left(\Phi^{(n)}-P_{\mathcal{B}^{(n)}} \operatorname{Cov}^{T}(\mathbf{X}, \mathbf{Y}) P_{\mathcal{A}^{(n)}}^{T} P_{\mathcal{A}^{(n)}} \operatorname{Cov}(\mathbf{X}, \mathbf{Y}) P_{\mathcal{B}^{(n)}}^{T}\right)\right\|_{F} \\
& \quad+\left\|\left(\phi^{(n)}-\delta\right) \cdot P_{\mathcal{B}^{(n)}} \operatorname{Cov}^{T}(\mathbf{X}, \mathbf{Y}) P_{\mathcal{A}^{(n)}}^{T} P_{\mathcal{A}^{(n)}} \operatorname{Cov}(\mathbf{X}, \mathbf{Y}) P_{\mathcal{B}^{(n)}}^{T}\right\|_{F} \rightarrow 0
\end{aligned}
$$


as $n \rightarrow \infty$. Thus, by Weyl's Theorem for Hermitian matrices, for each $i=1,2, \ldots, m$ we have almost surely that

$$
\underset{\quad}{\quad} \lambda_{i}\left[\phi^{(n)} \cdot \Phi^{(n)}\right]-\lambda_{i}\left[\delta \cdot\left(P_{\mathcal{A}^{(n)}} \operatorname{Cov}(\mathbf{X}, \mathbf{Y}) P_{\mathcal{B}^{(n)}}^{T}\right)^{T} P_{\mathcal{A}^{(n)}} \operatorname{Cov}(\mathbf{X}, \mathbf{Y}) P_{\mathcal{B}^{(n)}}^{T}\right] \mid
$$

as $n \rightarrow \infty$, from which Lemma 4 follows, after noting that $P_{\mathcal{B}^{(n)}}$ is symmetric.

We are now able to prove the main result of this section, Theorem 2.

Proof of Theorem 2: Let $\delta$ be as defined in Lemma 4. Note that for any nonnegative, bounded real sequences $\left\{a^{(n)}\right\}_{n=1}^{\infty}$ and $\left\{b^{(n)}\right\}_{n=1}^{\infty}$, it holds ${ }^{1}$ that $a^{(n)}-b^{(n)} \rightarrow 0$ if and only if $\sqrt{a^{(n)}}-\sqrt{b^{(n)}} \rightarrow 0$, as $n \rightarrow \infty$. Thus, by Lemma 4 , and noting that the rank of $P_{\mathcal{A}^{(n)}} \operatorname{Cov}(\mathbf{X}, \mathbf{Y}) P_{\mathcal{B}^{(n)}}$ is at most $k$, we have almost surely that, as $n \rightarrow \infty$,

$$
\sum_{i=1}^{m} \sigma_{i}\left(\mathcal{Y}^{(n)} \mathcal{X}^{(n) T}\right)-\sqrt{\delta} \cdot \sum_{i=1}^{k} \sigma_{i}\left(P_{\mathcal{A}^{(n)}} \operatorname{Cov}(\mathbf{X}, \mathbf{Y}) P_{\mathcal{B}^{(n)}}\right) \rightarrow 0 .
$$

But the expression in (8) can be simplified, by (5) and (3), as

$$
\begin{aligned}
2 k & -2 \sum_{i=1}^{m} \sigma_{i}\left(\mathcal{Y}^{(n)} \mathcal{X}^{(n) T}\right)-\left[2 k-2 \sqrt{\delta} \sum_{i=1}^{k} \sigma_{i}\left(P_{\mathcal{A}^{(n)}} \operatorname{Cov}(\mathbf{X}, \mathbf{Y}) P_{\mathcal{B}^{(n)}}\right)\right] \\
= & \epsilon^{2}\left(\mathcal{X}^{(n)}, \mathcal{Y}^{(n)}\right) \\
& -\left[2 k-2 \rho \sum_{i=1}^{k}\left(\frac{1}{\frac{1}{k} \sum_{j=1}^{k} \sigma_{j}(\operatorname{Cov}(\mathbf{X}, \mathbf{Y}))} \sigma_{i}\left(P_{\mathcal{A}^{(n)}} \operatorname{Cov}(\mathbf{X}, \mathbf{Y}) P_{\mathcal{B}^{(n)}}\right)\right)\right] \\
= & \epsilon^{2}\left(\mathcal{X}^{(n)}, \mathcal{Y}^{(n)}\right)-[(1-\rho) \cdot 2 k+ \\
& \left.\rho \cdot \sum_{i=1}^{k} 2\left(1-\frac{1}{\frac{1}{k} \sum_{j=1}^{k} \sigma_{j}(\operatorname{Cov}(\mathbf{X}, \mathbf{Y}))} \sigma_{i}\left(P_{\mathcal{A}^{(n)}} \operatorname{Cov}(\mathbf{X}, \mathbf{Y}) P_{\mathcal{B}^{(n)}}\right)\right)\right] \\
= & \epsilon^{2}\left(\mathcal{X}^{(n)}, \mathcal{Y}^{(n)}\right)-\left[(1-\rho) \cdot 2 k+\rho \cdot \partial^{2}\left(\mathcal{A}^{(n)}, \mathcal{B}^{(n)}\right)\right],
\end{aligned}
$$

which establishes Theorem 2.

1. Indeed, because $a^{(n)}$ and $b^{(n)}$ are bounded, and since $\left|a^{(n)}-b^{(n)}\right|=\left|\sqrt{a^{(n)}}-\sqrt{b^{(n)}}\right|$. $\left|\sqrt{a^{(n)}}+\sqrt{b^{(n)}}\right|$, we have that $\sqrt{a^{(n)}}-\sqrt{b^{(n)}} \rightarrow 0$ implies $a^{(n)}-b^{(n)} \rightarrow 0$. (Without the boundedness assumption this implication may not hold.) Conversely, if $\sqrt{a^{(n)}}-\sqrt{b^{(n)}} \nrightarrow 0$, then there exists $c>0$ such that $\left|\sqrt{a^{\left(n_{i}\right)}}-\sqrt{b^{\left(n_{i}\right)}}\right| \geq c$ for a subsequence, in which case $\left|a^{(n)}-b^{(n)}\right|=\left|\sqrt{a^{(n)}}-\sqrt{b^{(n)}}\right| \cdot\left|\sqrt{a^{(n)}}+\sqrt{b^{(n)}}\right| \geq c \cdot c$, hence $a^{(n)}-b^{(n)} \not \nrightarrow 0$. 
There is a special case of Theorem 2 that deserves attention:

Theorem 5. In the setting of Section 3.1, if $\operatorname{Cov}(\mathbf{X})=\operatorname{Cov}(\mathbf{Y})$ and $\operatorname{Cov}(\mathbf{X}, \mathbf{Y})=\beta I_{m}$ for a real number $\beta$, then it holds almost surely that

$$
\epsilon^{2}\left(\mathcal{X}^{(n)}, \mathcal{Y}^{(n)}\right)-\left[\left(1-\frac{|\beta|}{\alpha^{\prime}}\right) \cdot 2 k+\frac{|\beta|}{\alpha^{\prime}} \cdot d^{2}\left(\mathcal{A}^{(n)}, \mathcal{B}^{(n)}\right)\right] \rightarrow 0
$$

as $n \rightarrow \infty$, where $\alpha^{\prime}:=\frac{1}{k} \sum_{j=1}^{k} \sigma_{j}(\operatorname{Cov}(\mathbf{X}))$.

Theorem 5 is an immediate consequence of Theorem 2, since we previously pointed out that when $\operatorname{Cov}(\mathbf{X}, \mathbf{Y})$ is a scalar multiple of the identity then $\check{\partial}^{2}\left(\mathcal{A}^{(n)}, \mathcal{B}^{(n)}\right)=d^{2}\left(\mathcal{A}^{(n)}, \mathcal{B}^{(n)}\right)$.

Finally, Theorem 1 from Section 2 is an immediate consequence of Theorem 5, after noting that the setting of Section 2 is a special case of the setting of Section 3.1, with (recall the definitions of $\alpha$ and $\gamma$ from Section 2)

$$
\operatorname{Cov}\left[\begin{array}{c}
\mathbf{X} \\
\mathbf{Y}
\end{array}\right]=\left[\begin{array}{cr}
\alpha \cdot I_{m} & \gamma^{2} \cdot \alpha \cdot I_{m} \\
\gamma^{2} \cdot \alpha \cdot I_{m} & \alpha \cdot I_{m}
\end{array}\right] \in \mathbb{R}^{2 m \times 2 m}
$$

So $|\beta|$ and $\alpha^{\prime}$ of Theorem 5 are, respectively, $\gamma^{2} \cdot \alpha$ and $\alpha$, thus in Theorem 5 we have $\frac{|\beta|}{\alpha^{\prime}}=\frac{\gamma^{2} \cdot \alpha}{\alpha}=\gamma^{2}$. This proves Theorem 1 .

\subsection{Bounds for $ð^{2}$ and $\rho$}

Proposition 6. For $\check{\partial}^{2}\left(\mathcal{A}^{(n)}, \mathcal{B}^{(n)}\right)$ as defined in (3), it holds that $0 \leq$ $\varlimsup^{2}\left(\mathcal{A}^{(n)}, \mathcal{B}^{(n)}\right) \leq 2 k$.

Proof of Proposition 6: The upper bound is trivial. To prove the lower bound, first we re-express (3) as

$$
\begin{aligned}
& \partial^{2}\left(\mathcal{A}^{(n)}, \mathcal{B}^{(n)}\right)= \\
& \frac{2}{\frac{1}{k} \sum_{j=1}^{k} \sigma_{j}(\operatorname{Cov}(\mathbf{X}, \mathbf{Y}))} \sum_{i=1}^{k}\left(\sigma_{i}(\operatorname{Cov}(\mathbf{X}, \mathbf{Y}))-\sigma_{i}\left(P_{\mathcal{A}^{(n)}} \operatorname{Cov}(\mathbf{X}, \mathbf{Y}) P_{\mathcal{B}^{(n)}}\right)\right),
\end{aligned}
$$

and we show that each summand in the summation of (9) will be nonnegative. Indeed, for any $S \in \mathbb{R}^{m \times m}$ and $i=1,2, \ldots, n$, we have that $\sigma_{i}\left(S \cdot P_{\mathcal{A}^{(n)}}\right) \leq \sigma_{i}(S)$ and $\sigma_{i}\left(P_{\mathcal{A}^{(n)}} S\right) \leq \sigma_{i}(S)$; this is seen as follows. Say $P_{\mathcal{A}^{(n)}}=Q E Q^{T}$ is such that $Q \in \mathbb{R}^{m \times m}$ is orthogonal and $E$ is diagonal with 1's and 0's on its diagonal. Then $\sigma_{i}^{2}\left(S \cdot P_{\mathcal{A}^{(n)}}\right)=\lambda_{i}\left(P_{\mathcal{A}^{(n)}}^{T} S^{T} S P_{\mathcal{A}^{(n)}}\right)=$ 
$\lambda_{i}\left(Q E Q^{T} S^{T} S Q E Q^{T}\right)=\lambda_{i}\left(E Q^{T} S^{T} S Q E\right) \leq \lambda_{i}\left(Q^{T} S^{T} S Q\right)=\lambda_{i}\left(S^{T} S\right)$ $=\sigma_{i}^{2}(S)$, the inequality holding by the Interlacing Theorem for Hermitian matrices. By a similar argument $\sigma_{i}\left(P_{\mathcal{A}^{(n)}} S\right) \leq \sigma_{i}(S)$, and applying these in succession yields that $\sigma_{i}\left(P_{\mathcal{A}^{(n)}} \operatorname{Cov}(\mathbf{X}, \mathbf{Y}) P_{\mathcal{B}^{(n)}}\right) \leq \sigma_{i}(\operatorname{Cov}(\mathbf{X}, \mathbf{Y}))$.

Proposition 7. For $\rho$, as defined in Theorem 2, it holds that $0 \leq \rho \leq 1$.

Proof of Proposition 7. Let $\operatorname{Cov}(\mathbf{X}, \mathbf{Y})=U \Lambda V^{T}$ be a singular value decomposition; i.e. $U, V \in \mathbb{R}^{m \times m}$ are orthogonal and $\Lambda \in \mathbb{R}^{m \times m}$ is diagonal, with nonincreasing nonnegative diagonal entries. Define $M \in \mathbb{R}^{2 m \times 2 m}$ by

$$
\begin{aligned}
M & :=\left[\begin{array}{ll}
U^{T} & 0_{m} \\
0_{m} & V^{T}
\end{array}\right]\left[\begin{array}{cc}
\operatorname{Cov}(\mathbf{X}) & \operatorname{Cov}(\mathbf{X}, \mathbf{Y}) \\
\operatorname{Cov}^{T}(\mathbf{X}, \mathbf{Y}) & \operatorname{Cov}(\mathbf{Y})
\end{array}\right]\left[\begin{array}{cc}
U & 0_{m} \\
0_{m} & V
\end{array}\right] \\
& =\left[\begin{array}{cc}
U^{T} \operatorname{Cov}(\mathbf{X}) U & \Lambda \\
\Lambda & V^{T} \operatorname{Cov}(\mathbf{Y}) V
\end{array}\right]
\end{aligned}
$$

where $0_{m} \in \mathbb{R}^{m \times m}$ is the matrix of zeros. A covariance matrix is positive semidefinite, thus $M$ is positive semidefinite, as well as all of its principal submatrices. For each $j=1,2, \ldots, k$, the two-by-two submatrix consisting of the $j$ th and $j+m$ th rows and columns of $M$ has nonnegative diagonals and a nonnegative determinant, thus $\left(U^{T} \operatorname{Cov}(\mathbf{X}) U\right)_{j j}\left(V^{T} \operatorname{Cov}(\mathbf{Y}) V\right)_{j j} \geq\left(\Lambda_{j j}\right)^{2}$, i.e.

$$
\sigma_{j}(\operatorname{Cov}(\mathbf{X}, \mathbf{Y})) \leq \sqrt{\left(U^{T} \operatorname{Cov}(\mathbf{X}) U\right)_{j j}} \cdot \sqrt{\left(V^{T} \operatorname{Cov}(\mathbf{Y}) V\right)_{j j}} \cdot
$$

Now, summing (10) over $j=1,2, \ldots, k$ and applying the Cauchy-Schwartz inequality to the resulting right-hand side, we obtain

$$
\sum_{j=1}^{k} \sigma_{j}(\operatorname{Cov}(\mathbf{X}, \mathbf{Y})) \leq \sqrt{\sum_{j=1}^{k}\left(U^{T} \operatorname{Cov}(\mathbf{X}) U\right)_{j j}} \cdot \sqrt{\sum_{j=1}^{k}\left(V^{T} \operatorname{Cov}(\mathbf{Y}) V\right)_{j j}}
$$

For any Hermitian matrix, the vector of its diagonals always majorizes the vector of its eigenvalues, thus

$$
\sum_{j=1}^{k}\left(U^{T} \operatorname{Cov}(\mathbf{X}) U\right)_{j j} \leq \sum_{j=1}^{k} \lambda_{j}\left(U^{T} \operatorname{Cov}(\mathbf{X}) U\right)=\sum_{j=1}^{k} \sigma_{j}(\operatorname{Cov}(\mathbf{X})),
$$

and Proposition 7 follows from (11), (12), and (12) applied to $\operatorname{Cov}(\mathbf{Y})$ and $V$. 


\subsection{An Isometry-Corrective Property of $\check{\partial}^{2}$}

Suppose that $W \in \mathbb{R}^{m \times m}$ is an orthogonal matrix such that

$$
\operatorname{Cov}\left[\begin{array}{r}
\mathbf{X} \\
W \mathbf{Y}
\end{array}\right]=\left[\begin{array}{cc}
\operatorname{Cov}(\mathbf{X}) & \beta \cdot I_{m} \\
\beta \cdot I_{m} & \operatorname{Cov}(\mathbf{X})
\end{array}\right] \in \mathbb{R}^{2 m \times 2 m},
$$

where $\beta \in \mathbb{R}$ is nonzero; this might arise in situations similar to the cautionary Tale of Two Scientists in Section 2-wherein two scientists are taking measurements of the same random process - except that the second scientist permutes the order of the features (i.e., $W$ is a permutation matrix). Define $W \mathcal{B}^{(n)}:=\left\{W x: x \in \mathcal{B}^{(n)}\right\}$. In this situation, the quantity $d^{2}\left(\mathcal{A}^{(n)}, W \mathcal{B}^{(n)}\right)$ may be more interesting than the quantity $d^{2}\left(\mathcal{A}^{(n)}, \mathcal{B}^{(n)}\right)$, since $\mathcal{A}^{(n)}$ might be viewed as being more comparable to $W \mathcal{B}^{(n)}$ then to $\mathcal{B}^{(n)}$. Indeed, if the eigenvalues of $\operatorname{Cov}(\mathbf{X})$ are distinct and $n$ is large and $W$ is not $I_{m}$, then $d^{2}\left(\mathcal{A}^{(n)}, W \mathcal{B}^{(n)}\right)$ would be small, in contrast to $d^{2}\left(\mathcal{A}^{(n)}, \mathcal{B}^{(n)}\right)$.

Proposition 8. In the case of the previous paragraph, we have $\mathrm{\partial}^{2}\left(\mathcal{A}^{(n)}, \mathcal{B}^{(n)}\right)$ $=d^{2}\left(\mathcal{A}^{(n)}, W \mathcal{B}^{(n)}\right)$.

Proposition 8 will be illustrated in Section 4.3.

Proof of Proposition 8. Here we have

$$
\begin{aligned}
\operatorname{Cov}\left[\begin{array}{l}
\mathbf{X} \\
\mathbf{Y}
\end{array}\right] & =\left[\begin{array}{cc}
I_{m} & 0_{m} \\
0_{m} & W^{T}
\end{array}\right]\left[\begin{array}{cc}
\operatorname{Cov}(\mathbf{X}) & \beta \cdot I_{m} \\
\beta \cdot I_{m} & \operatorname{Cov}(\mathbf{X})
\end{array}\right]\left[\begin{array}{cc}
I_{m} & 0_{m} \\
0_{m} & W
\end{array}\right] \\
& =\left[\begin{array}{cc}
\operatorname{Cov}(\mathbf{X}) & \beta \cdot W \\
\beta \cdot W^{T} & \operatorname{Cov}(\mathbf{Y})
\end{array}\right],
\end{aligned}
$$

thus for all $i=1,2, \ldots, m$

$$
\begin{gathered}
\sigma_{i}(\operatorname{Cov}(\mathbf{X}, \mathbf{Y}))=\sigma_{i}(\beta \cdot W)=|\beta| \\
\text { and } \\
\sigma_{i}\left(P_{\mathcal{A}^{(n)}} \operatorname{Cov}(\mathbf{X}, \mathbf{Y}) P_{\mathcal{B}^{(n)}}\right)=|\beta| \cdot \sigma_{i}\left(P_{\mathcal{A}^{(n)}} W P_{\mathcal{B}^{(n)}}\right) \\
=|\beta| \cdot \sigma_{i}\left(P_{\mathcal{A}^{(n)}} W P_{\mathcal{B}^{(n)}} W^{T}\right)
\end{gathered}
$$

Because $P_{W \mathcal{B}^{(n)}}=W P_{\mathcal{B}^{(n)}} W^{T}$, and by (2), (3), (13) and (14), it follows that $\partial^{2}\left(\mathcal{A}^{(n)}, \mathcal{B}^{(n)}\right)=\sum_{i=1}^{k} 2\left(1-\sigma_{i}\left(P_{\mathcal{A}^{(n)}} W P_{\mathcal{B}^{(n)}} W^{T}\right)\right)=d^{2}\left(\mathcal{A}^{(n)}, W \mathcal{B}^{(n)}\right)$. 


\section{Simulations and Real Data}

In this section, simulations and real data illustrate and support the theorems which we stated and proved in the previous sections, and we then use these simulations and real data to illustrate how the "incommensurability phenomenon" can arise as a consequence. What is meant by this phenomenon is the occurrence of an inordinately large Procrustean fitting-error between projected data that was originally highly-correlated. (This phenomenon was named in Priebe, Marchette, Ma, and Adali 2013).

\subsection{A First Illustration}

Our first illustration of Theorem 2 and Theorem 5 is with $\mathbf{X}$ and $\mathbf{Y}$ distributed multivariate normal (with mean vector consisting of all zeros) such that $\operatorname{Cov}(\mathbf{X})=\operatorname{Cov}(\mathbf{Y})=I_{6}$ and $\operatorname{Cov}(\mathbf{X}, \mathbf{Y})=\beta \cdot I_{6}$ for assorted values of $\beta$. Note that $\rho$ as defined in Theorem 2 is $\beta$ here, note that $\alpha^{\prime}$ and $\beta$ as defined in Theorem 5 are, respectively, 1 and $\beta$ here, and note that here $\partial^{2}\left(\mathcal{A}^{(n)}, \mathcal{B}^{(n)}\right)=d^{2}\left(\mathcal{A}^{(n)}, \mathcal{B}^{(n)}\right)$ because $\operatorname{Cov}(\mathbf{X}, \mathbf{Y})$ is a scalar multiple of the identity. Also, this example may be seen as an illustration of Theorem 1 -in the Tale of Two Scientists—with $\gamma^{2}$ there being $\beta$ here.

The dimension of the space containing $\mathbf{X}$ and $\mathbf{Y}$ is $m=6$, and we will project to spaces of dimension $k=2$.

For each of $\beta=0, .1, .2, .3, .4, .5, .6, .7, .8, .9, .99$, and for each of $n=1000$ and $n=10000$ we obtained 1000 realizations of $\mathcal{X}^{(n)}$ and $\mathcal{Y}^{(n)}$ and used PCA to obtain $\mathcal{A}^{(n)}$ and $\mathcal{B}^{(n)}$. In Figure 1, we plotted the values of $\epsilon^{2}\left(\mathcal{X}^{(n)}, \mathcal{Y}^{(n)}\right)$ against the respective values of $d^{2}\left(\mathcal{A}^{(n)}, \mathcal{B}^{(n)}\right)$, in colors blue, green, red, cyan, magenta, blue, green, red, cyan, magenta, blue for the respective values of $\beta=0, .1, .2, .3, .4, .5, .6, .7, .8, .9, .99$. For reference, we also included -in Figure 1 -lines with y-intercept $(1-\beta) \cdot 2 k$ and slope $\beta$, for each of the above-specified values of $\beta$; basically, Theorem 1 , Theorem 2, and Theorem 5 state that the scatter plots will adhere to these respective lines in the limit as $n$ goes to $\infty$. Indeed, notice in Figure 1 that the scatter plots adhere very closely to their respective lines, and such adherence substantially improves as $n=1000$ is raised to $n=10000$, which supports/illustrates the claims of Theorem 1, Theorem 2, and Theorem 5.

The above was done using PCA to generate $\mathcal{A}^{(n)}$ and $\mathcal{B}^{(n)}$. What if we instead took $\mathcal{A}^{(n)}$ and $\mathcal{B}^{(n)}$ to (each) be the span of the first two standardbasis vectors in $\mathbb{R}^{6}$ ? We will call this the "trivial" choice of $\mathcal{A}^{(n)}$ and $\mathcal{B}^{(n)}$. Of course, the value of $d^{2}\left(\mathcal{A}^{(n)}, \mathcal{B}^{(n)}\right)$ would always be identically zero, and note that Theorem 1, Theorem 2, and Theorem 5 still apply with this choice of $\mathcal{A}^{(n)}$ and $\mathcal{B}^{(n)}$ because $\operatorname{Cov}(\mathbf{X})$ and $\operatorname{Cov}(\mathbf{Y})$ are scalar multiples of the identity. Thus, the scatter plots from these above experiments when they are 

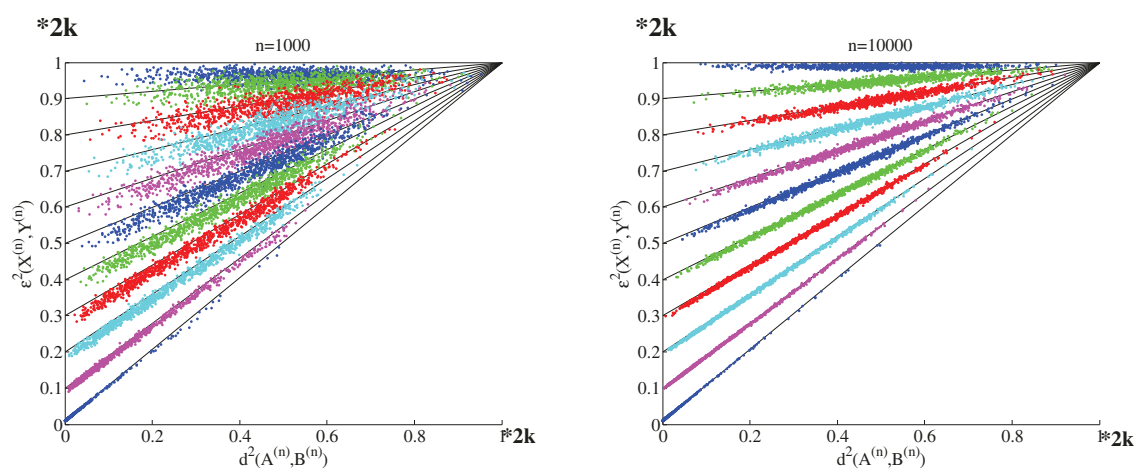

Figure 1. Plots of $\epsilon^{2}\left(\mathcal{X}^{(n)}, \mathcal{Y}^{(n)}\right)$ vs $d^{2}\left(\mathcal{A}^{(n)}, \mathcal{B}^{(n)}\right)$ when $\operatorname{Cov}(\mathbf{X})=\operatorname{Cov}(\mathbf{Y})=I_{6}$, $\operatorname{Cov}(\mathbf{X}, \mathbf{Y})=\beta \cdot I_{6}$. For each of $\beta=0$ (blue), .1 (green), .2 (red), .3 (cyan), .4 (magenta), .5 (blue), .6 (green), .7 (red), .8 (cyan), .9 (magenta), .99 (blue), for each of $n=1000$ (left) and $n=10000$ (right), there were 1000 Monte Carlo replicates using $k=2$. Note that the axis-values are to be multiplied by $2 k$, which is 4 here, since the ranges of $\epsilon^{2}\left(\mathcal{X}^{(n)}, \mathcal{Y}^{(n)}\right)$ and $d^{2}\left(\mathcal{A}^{(n)}, \mathcal{B}^{(n)}\right)$ are the interval $[0,2 k]$.

performed instead for the trivial choice of $\mathcal{A}^{(n)}$ and $\mathcal{B}^{(n)}$ would land in the far left of Figure 1 (along the y-axis at $d^{2}\left(\mathcal{A}^{(n)}, \mathcal{B}^{(n)}\right)=0$ ), clustered about their respective lines. Indeed, we then performed the above experiments for the trivial choice of $\mathcal{A}^{(n)}$ and $\mathcal{B}^{(n)}$; the sample mean and sample standard deviation of $\epsilon^{2}\left(\mathcal{X}^{(n)}, \mathcal{Y}^{(n)}\right)$ for the 1000 Monte Carlo replicates when $n=$ 10000 were as follows:

\begin{tabular}{c||c|c} 
& $\begin{array}{c}\text { mean, } \\
\text { st.dev. of } \epsilon^{2}\left(\mathcal{X}^{(\mathrm{n})}, \mathcal{Y}^{(\mathrm{n})}\right) \text { with PCA }\end{array}$ & $\begin{array}{c}\text { mean, } \\
\text { st.dev. of } \epsilon^{2}\left(\mathcal{X}^{(\mathrm{n})}, \mathcal{Y}^{(\mathrm{n})}\right) \text { with } \\
\text { trivial } \mathcal{A}^{(\mathrm{n})} \text { and } \mathcal{B}^{(\mathrm{n})}\end{array}$ \\
\hline$\beta=0$ & $3.9546,0.0170$ & $3.9534,0.0178$ \\
$\beta=.1$ & $3.7903,0.0618$ & $3.6003,0.0277$ \\
$\beta=.2$ & $3.5774,0.1179$ & $3.1994,0.0276$ \\
$\beta=.3$ & $3.3413,0.1796$ & $2.7990,0.0254$ \\
$\beta=.4$ & $3.0942,0.2429$ & $2.4006,0.0230$ \\
$\beta=.5$ & $2.7918,0.3043$ & $1.9999,0.0210$ \\
$\beta=.6$ & $2.4581,0.3658$ & $1.5996,0.0177$ \\
$\beta=.7$ & $2.0331,0.4283$ & $1.2007,0.0140$ \\
$\beta=.8$ & $1.5368,0.4567$ & $0.8003,0.0103$ \\
$\beta=.9$ & $0.9232,0.4607$ & $0.4001,0.0054$ \\
$\beta=.99$ & $0.1352,0.2057$ & $0.0400,0.0006$
\end{tabular}

Indeed, besides the notable exception when $\beta=0$ (where there is no correlation anyway between $\mathbf{X}$ and $\mathbf{Y})$, the values of $\epsilon^{2}\left(\mathcal{X}^{(n)}, \mathcal{Y}^{(n)}\right)$ were substantially larger when PCA was used to generate $\mathcal{A}^{(n)}$ and $\mathcal{B}^{(n)}$ than for the trivial choice of $\mathcal{A}^{(n)}$ and $\mathcal{B}^{(n)}$. This is the incommensurability phenomenon, a situation where use of PCA has the consequence of inordinately large Procrustean fitting-error. 
Let us call the values $\epsilon^{2}\left(\mathcal{X}^{(n)}, \mathcal{Y}^{(n)}\right)-\left[(1-\beta) \cdot 2 k+\beta \cdot d^{2}\left(\mathcal{A}^{(n)}, \mathcal{B}^{(n)}\right)\right]$ residuals. It is noteworthy that in the above experiments the sample standard deviation of the residuals when PCA was used to generate $\mathcal{A}^{(n)}$ and $\mathcal{B}^{(n)}$ is very close to the sample standard deviation of $\epsilon^{2}\left(\mathcal{X}^{(n)}, \mathcal{Y}^{(n)}\right)$ for the trivial choice of $\mathcal{A}^{(n)}$ and $\mathcal{B}^{(n)}$. Specifically, we computed:

\begin{tabular}{c||c|c} 
& st.dev. of $\epsilon^{2}\left(\mathcal{X}^{(\mathrm{n})}, \mathcal{Y}^{(\mathrm{n})}\right)$ with PCA & $\begin{array}{c}\text { st.dev. of } \epsilon^{2}\left(\mathcal{X}^{(\mathrm{n})}, \mathcal{Y}^{(\mathrm{n})}\right) \text { with } \\
\operatorname{trivial} \mathcal{A}^{(\mathrm{n})} \text { and } \mathcal{B}^{(\mathrm{n})}\end{array}$ \\
\hline$\beta=0$ & 0.0170 & 0.0178 \\
$\beta=.1$ & 0.0267 & 0.0277 \\
$\beta=.2$ & 0.0262 & 0.0276 \\
$\beta=.3$ & 0.0252 & 0.0254 \\
$\beta=.4$ & 0.0235 & 0.0230 \\
$\beta=.5$ & 0.0214 & 0.0210 \\
$\beta=.6$ & 0.0192 & 0.0177 \\
$\beta=.7$ & 0.0158 & 0.0140 \\
$\beta=.8$ & 0.0118 & 0.0103 \\
$\beta=.9$ & 0.0073 & 0.0054 \\
$\beta=.99$ & 0.0025 & 0.0006
\end{tabular}

So, it seems empirically here that the variation in $\epsilon^{2}\left(\mathcal{X}^{(n)}, \mathcal{Y}^{(n)}\right)$ not explained by $d^{2}\left(\mathcal{A}^{(n)}, \mathcal{B}^{(n)}\right)$ when PCA generates $\mathcal{A}^{(n)}$ and $\mathcal{B}^{(n)}$ is approximately the same as the variation in $\epsilon^{2}\left(\mathcal{X}^{(n)}, \mathcal{Y}^{(n)}\right)$ for the trivial choice of $\mathcal{A}^{(n)}$ and $\mathcal{B}^{(n)}$ (in which $d^{2}\left(\mathcal{A}^{(n)}, \mathcal{B}^{(n)}\right)=0$ identically) and, as such, $d^{2}\left(\mathcal{A}^{(n)}, \mathcal{B}^{(n)}\right)$ explains all of the rest of the variation here in $\epsilon^{2}\left(\mathcal{X}^{(n)}, \mathcal{Y}^{(n)}\right)$ when PCA is used.

\subsection{A Second Illustration}

Our next illustration of Theorem 2 and Theorem 5 is with $\mathbf{X}$ and $\mathbf{Y}$ multivariate normal (with mean vector of all zeros) such that $\operatorname{Cov}(\mathbf{X})=$ $\operatorname{Cov}(\mathbf{Y})=$ the diagonal matrix in $\mathbb{R}^{20 \times 20}$ with .7 on all diagonals except for the first diagonal, which has the value 1 , and such that $\operatorname{Cov}(\mathbf{X}, \mathbf{Y})=.6 * I_{20}$. So we are using $m=20$ here. As above, $\partial^{2}\left(\mathcal{A}^{(n)}, \mathcal{B}^{(n)}\right)=d^{2}\left(\mathcal{A}^{(n)}, \mathcal{B}^{(n)}\right)$ because $\operatorname{Cov}(\mathbf{X}, \mathbf{Y})$ is a scalar multiple of the identity.

We will use three different projection dimensions, each of $k=1,2,10$. When $k=1$ the formula in Theorem 2 yields $\rho=\frac{.6}{1}=.6$, when $k=2$ the formula yields $\rho=\frac{.6+.6}{1+.7} \approx .7059$, and when $k=10$ the formula yields $\rho=\frac{.6+.6+.6 \cdots}{1+.7+.7 \cdots} \approx .8219$.

Using PCA to generate $\mathcal{A}^{(n)}$ and $\mathcal{B}^{(n)}$, we obtained 10000 realizations of $\mathcal{X}^{(n)}$ and $\mathcal{Y}^{(n)}$ when $n=10000$, for each projection dimension $k=1$, $k=2$, and $k=10$; the values of $\epsilon^{2}\left(\mathcal{X}^{(n)}, \mathcal{Y}^{(n)}\right)$ are plotted against the respective values of $d^{2}\left(\mathcal{A}^{(n)}, \mathcal{B}^{(n)}\right)$ in the left figure of Figure 2, with $k=1$ in blue, $k=2$ in red, and $k=10$ in green. As before, lines are drawn on 

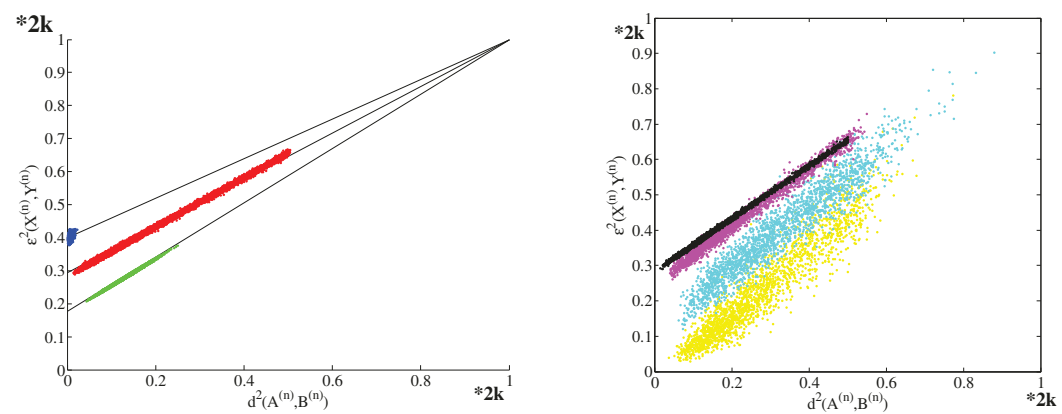

Figure 2. Plots of $\epsilon^{2}\left(\mathcal{X}^{(n)}, \mathcal{Y}^{(n)}\right)$ vs $d^{2}\left(\mathcal{A}^{(n)}, \mathcal{B}^{(n)}\right)$ for $\operatorname{Cov}(\mathbf{X})=\operatorname{Cov}(\mathbf{Y})=$ $\operatorname{diag}(1, .7, .7, \ldots, .7) \in \mathbb{R}^{20 \times 20}, \operatorname{Cov}(\mathbf{X}, \mathbf{Y})=.6 * I_{20}$. The figure on the left shows 10000 Monte Carlo replications when $n=10000$, for each of $k=1$ (blue), $k=2$ (red), and $k=10$ (green). The figure on the right shows 2000 Monte Carlo replications when $k=2$, for each of $n=10^{1}$ (yellow), $n=10^{2}$ (cyan), $n=10^{3}$ (magenta), and $n=10^{4}$ (black). Note that the axis-values are to be multiplied by $2 k$ for the respective values of $k$, since the ranges of $\epsilon^{2}\left(\mathcal{X}^{(n)}, \mathcal{Y}^{(n)}\right)$ and $d^{2}\left(\mathcal{A}^{(n)}, \mathcal{B}^{(n)}\right)$ are the interval $[0,2 k]$.

the figure to indicate the limiting relationship between $\epsilon^{2}\left(\mathcal{X}^{(n)}, \mathcal{Y}^{(n)}\right)$ and $d^{2}\left(\mathcal{A}^{(n)}, \mathcal{B}^{(n)}\right)$ that is predicted by Theorem 2 and Theorem 5 ; indeed, the scatter plots adhere very closely to these respective lines. In the right hand side of Figure 2 is 2000 Monte Carlo simulations when $k=2$ for each of $n=10^{1}$ (yellow), $n=10^{2}$ (cyan), $n=10^{3}$ (magenta) and $n=10^{4}$ (black). As $n$ is getting larger, these are seen to get increasingly closer to the corresponding limiting relationship between $\epsilon^{2}\left(\mathcal{X}^{(n)}, \mathcal{Y}^{(n)}\right)$ and $d^{2}\left(\mathcal{A}^{(n)}, \mathcal{B}^{(n)}\right)$. All of this supports the claims of Theorem 2 and Theorem 5.

In the experiments for the left figure in Figure 2, the sample mean and sample standard deviation of $\frac{\epsilon^{2}\left(\mathcal{X}^{(n)}, \mathcal{Y}^{(n)}\right)}{2 k}$ were as follows:

\begin{tabular}{c||c|c} 
& sample mean of $\frac{\epsilon^{2}\left(\mathcal{X}^{(n)}, \mathcal{Y}^{(n)}\right)}{2 k}$ & sample standard deviation of $\frac{\epsilon^{2}\left(\mathcal{X}^{(n)}, \mathcal{Y}^{(n)}\right)}{2 k}$ \\
\hline$k=1$ & .4017 & .0069 \\
$k=2$ & .4323 & .0950 \\
$k=10$ & .2797 & .0244
\end{tabular}

We normalize $\epsilon^{2}\left(\mathcal{X}^{(n)}, \mathcal{Y}^{(n)}\right)$ with division by $2 k$ since the range of $\epsilon^{2}\left(\mathcal{X}^{(n)}\right.$, $\left.\mathcal{Y}^{(n)}\right)$ is $[0,2 k]$. As $k$ increases, the correlation $\rho$ increases, so it would seem at first thought that the normalized Procrustean fitting-error $\frac{\epsilon^{2}\left(\mathcal{X}^{(n)}, \mathcal{Y}^{(n)}\right)}{2 k}$ should decrease. Indeed, the leftmost green points in (the left figure of) Figure 2 are below the leftmost red points, which are below the leftmost blue points. However, overall, the normalized Procrustean fitting-error is seen in the table above to be much higher in the case of $k=2$ than the case of $k=1$. This is explained by noting a substantial gap between the first eigenvalue of $\operatorname{Cov}(\mathbf{X})$ and the second eigenvalue of $\operatorname{Cov}(\mathbf{X})$ (1 vs .7) whereas there is no gap between the second eigenvalue of of $\operatorname{Cov}(\mathbf{X})$ and 
the third eigenvalue of $\operatorname{Cov}(\mathbf{X})$ (both are .7). Thus when $k=1$ the PCA projection has little variance whereas when $k=2$ the PCA projection has much variance, often causing much larger Hausdorff distance between $\mathcal{A}^{(n)}$ and $\mathcal{B}^{(n)}$, which results in larger Procrustean fitting-error by Theorem 2. As such, the case of $k=2$ is an example of the incommensurability phenomenon of inordinately large Procrustean fitting-error. But then observe that when $k=10$ we find that the normalized Procrustean fitting-error is competitive with the $k=1$ case; even though the tenth and eleventh eigenvalues of $\operatorname{Cov}(\mathbf{X})$ are the same, nonetheless the correlation $\rho$ has increased, and the variance of the PCA projection has decreased enough to improve the normalized Procrustean fitting-error to be competitive with the case of $k=1$.

Not only may the incommensurability phenomenon occur when there is no spectral gap in the covariance structure at the projection dimension, but the incommensurability phenomenon may occur when this spectral gap is positive but small. Indeed, repeating the experiments performed for the left figure in Figure 2, and just changing the second diagonal of $\operatorname{Cov}(\mathbf{X})=$ $\operatorname{Cov} \mathbf{Y}$ ) from .7 to $\lambda$ for each of $\lambda=.71, .72, .73, .74, .75$ but otherwise the experiments are the same, we got a very similar-looking scatter plot as the left figure in Figure 2, and the sample mean and sample standard deviation of $\frac{\epsilon^{2}\left(\mathcal{X}^{(n)}, \mathcal{Y}^{(n)}\right)}{2 k}$ were as follows:

\begin{tabular}{|c|c|c|c|}
\hline & $k=1$ & $k=2$ & $k=10$ \\
\hline sample mean of $\frac{\epsilon^{2}\left(\mathcal{X}^{(n)}, \mathcal{Y}^{(n)}\right)}{2 k}$ when $\lambda=.71$ & .4017 & .4342 & .2807 \\
\hline sample mean of $\frac{\epsilon^{2}\left(\mathcal{X}^{(n)}, \mathcal{Y}^{(n)}\right)}{2 k}$ when $\lambda=.72$ & .4018 & .4329 & .2809 \\
\hline sample mean of $\frac{\epsilon^{2}\left(\mathcal{X}^{(n)}, \mathcal{Y}^{(n)}\right)}{2 k}$ when $\lambda=.73$ & .4018 & .4207 & .2810 \\
\hline sample mean of $\frac{\epsilon^{2}\left(\mathcal{X}^{(n)}, \mathcal{Y}^{(n)}\right)}{2 k}$ when $\lambda=.74$ & .4018 & .3997 & .2815 \\
\hline sample mean of $\frac{\epsilon^{2}\left(\mathcal{X}^{(n)}, \mathcal{Y}^{(n)}\right)}{2 k}$ when $\lambda=.75$ & .4019 & .3754 & .2815 \\
\hline sample stdev of $\frac{\epsilon^{2}\left(\mathcal{X}^{(n)}, \mathcal{Y}^{(n)}\right)}{2 k}$ when $\lambda=.71$ & .0068 & .0960 & .0244 \\
\hline sample stdev of $\frac{\epsilon^{2}\left(\mathcal{X}^{(n)}, \mathcal{Y}^{(n)}\right)}{2 k}$ when $\lambda=.72$ & .0070 & .0953 & .0243 \\
\hline sample stdev of $\frac{\epsilon^{2}\left(\mathcal{X}^{(n)}, \mathcal{Y}^{(n)}\right)}{2 k}$ when $\lambda=.73$ & .0069 & .0922 & .0245 \\
\hline sample stdev of $\frac{\epsilon^{2}\left(\mathcal{X}^{(n)}, \mathcal{Y}^{(n)}\right)}{2 k}$ when $\lambda=.74$ & .0070 & .0832 & .0244 \\
\hline sample stdev of $\frac{\epsilon^{2}\left(\mathcal{X}^{(n)}, \mathcal{Y}^{(n)}\right)}{2 k}$ when $\lambda=.75$ & .0070 & .0662 & .0242 \\
\hline
\end{tabular}

In the case of $k=2$, the spectral gap in the covariance structure at the projection dimension is $\lambda-.7$, and note that as this gap grows to $.75-$ $.7=.05$ there is a lessening of the incommensurability phenomenon, but the phenomenon is still very much present. Indeed (from the table above), when $\lambda=.75$, the sample mean of $\frac{\epsilon^{2}\left(\mathcal{X}^{(n)}, \mathcal{Y}^{(n)}\right)}{2 k}$ when $k=2$ (see table above) is below the sample mean when $k=1$, but it is only lower by less than a half of the sample standard deviation of $\frac{\epsilon^{2}\left(\mathcal{X}^{(n)}, \mathcal{Y}^{(n)}\right)}{2 k}$ when $k=2$ and, in fact, notice that the sample standard deviation of $\frac{\epsilon^{2}\left(\mathcal{X}^{(n)}, \mathcal{Y}^{(n)}\right)}{2 k}$ when $k=2$ 
is more than 9 times the sample standard deviation when $k=1$. Thus there is a significant probability of an inordinately high Procrustean fitting error in the case of $k=2$ with $\lambda=.75$.

\subsection{A Modification of the Second Illustration to Illustrate the Isometry- Corrective Property of $\partial^{2}$}

Our next illustration of Theorem 2 is with $\mathbf{X}$ and $\mathbf{Y}$ distributed multivariate normal, with joint covariance matrix given by:

$$
\operatorname{Cov}\left[\begin{array}{c}
\mathbf{X} \\
\mathbf{Y}
\end{array}\right]=\left[\begin{array}{cccccccccccc}
1 & 0 & 0 & \ldots & 0 & 0 & 0 & 0 & \ldots & 0 & 0 & .6 \\
0 & .7 & 0 & \ldots & 0 & 0 & 0 & 0 & \ldots & 0 & .6 & 0 \\
0 & 0 & .7 & \ldots & 0 & 0 & 0 & 0 & \ldots & .6 & 0 & 0 \\
\vdots & \vdots & \vdots & \ddots & \vdots & \vdots & \vdots & \vdots & & \vdots & \vdots & \vdots \\
0 & 0 & 0 & \ldots & .7 & 0 & 0 & .6 & \ldots & 0 & 0 & 0 \\
0 & 0 & 0 & \ldots & 0 & .7 & .6 & 0 & \ldots & 0 & 0 & 0 \\
0 & 0 & 0 & \ldots & 0 & .6 & .7 & 0 & \ldots & 0 & 0 & 0 \\
0 & 0 & 0 & \ldots & .6 & 0 & 0 & .7 & \ldots & 0 & 0 & 0 \\
\vdots & \vdots & \vdots & & \vdots & \vdots & \vdots & \vdots & \ddots & \vdots & \vdots & \vdots \\
0 & 0 & .6 & \ldots & 0 & 0 & 0 & 0 & \ldots & .7 & 0 & 0 \\
0 & .6 & 0 & \ldots & 0 & 0 & 0 & 0 & \ldots & 0 & .7 & 0 \\
.6 & 0 & 0 & \ldots & 0 & 0 & 0 & 0 & \ldots & 0 & 0 & 1
\end{array}\right] \in \mathbb{R}^{40 \times 40}
$$

Of course, this is exactly the illustration in the beginning of Section 4.2, with the only exception that the coordinates of $Y$ have been permuted into reverse order. Performing the very same experiments from the beginning of Section 4.2, the scatter plots of $\epsilon^{2}\left(\mathcal{X}^{(n)}, \mathcal{Y}^{(n)}\right)$ vs $d^{2}\left(\mathcal{A}^{(n)}, \mathcal{B}^{(n)}\right)$ will not look like the scatter plots in Figure 2. However, since the permutation transformation is an isometry, we then have by Proposition 8 in Section 3.4, that the scatter plots of $\epsilon^{2}\left(\mathcal{X}^{(n)}, \mathcal{Y}^{(n)}\right)$ vs $\partial^{2}\left(\mathcal{A}^{(n)}, \mathcal{B}^{(n)}\right)$ will indeed look like the scatter plots in Figure 2. The use of $\check{\partial}^{2}$ automatically accounts for isometrical transformations of $\mathbf{X}$ and/or $\mathbf{Y}$ from a common frame, in the manner of this example.

It should also be mentioned that, for the illustration of this section (with the covariance matrix above), if $\mathcal{A}^{(n)}$ and $\mathcal{B}^{(n)}$ were not generated with PCA, but instead $\mathcal{A}^{(n)}$ and $\mathcal{B}^{(n)}$ were selected to be (the same as each other by setting them to be) the span of any number of standard-basis vectors in $\mathbb{R}^{40}$ then the Procrustes fitting-error would be disasterously large. The fact that such a naive choice of $\mathcal{A}^{(n)}$ and $\mathcal{B}^{(n)}$ was successful in the illustration in Section 4.1 was just a byproduct of the good fortune that $\mathbf{X}$ and $\mathbf{Y}$ did not have permuted coordinates or any other isometrical transformation applied to them. 


\subsection{The Incommensurability Phenomenon in Real Data}

We next illustrate the incommensurability phenomenon using real data from the 2014 Science article of Vogelstein et. al., titled "Discovery of Brainwide Neural-Behavioral Maps Via Multiscale Unsupervised Structure Learning". See O'Leary and Marder (2014) for a big-picture overview and discussion of the contributions of this article. This data from Vogelstein et. al. will be observed by Two Scientists who will record highly correlated observations. We will show the incommensurability phenomenon creeping into the Two Scientist's efforts. (The data related to this section is available online at http://www.cis.jhu.edu/ parky/Incomm/.)

In the Vogelstein et. al. paper (2014), the authors consider a collection of optogenetically manipulated Drosophila larvae, with the goal of generating a behavioral reference atlas. The animals considered are partitioned into lines, with each line defined by the neuron classes which are being optogenetically manipulated. Each line includes multiple replicates-dishes-in each of which numerous animals are found. Videos of animal behavior are processed into a multivariate behavioral time series for each animal, these time series give rise to an animal dissimilarity matrix, and multidimensional scaling applied to this dissimilarity matrix yields a representation of the collection of animals in high-dimensional Euclidean space. For our purposes, we will focus our attention on the sixteen most significant dimensions; in this manner, every animal corresponds to a vector in $\mathbb{R}^{16}$.

In our experiment here, for each of $i=1,2,3, \ldots, 242$, the first scientist will record $\mathbf{X}^{(i)} \in \mathbb{R}^{16}$ and the second scientist will record $\mathbf{Y}^{(i)} \in$ $\mathbb{R}^{16}$, as follows. There were a total of $n=242$ dishes in the Vogelstein et. al. data set corresponding to the control line $p B D P U-C h R 2$. For each $i=1,2,3, \ldots, 242$, we select the two most correlated animals in the $i$ 'th dish, and the first scientist picks—equiprobably—one of these two animals, and sets $\mathbf{X}^{(i)} \in \mathbb{R}^{16}$ to be this animal's associated vector, and the other scientist is left with the other animal, and sets $\mathbf{Y}^{(i)} \in \mathbb{R}^{16}$ to be that animal's associated vector. The first scientist's observations are stored in the ma$\operatorname{trix} X^{(242)}=\left[\mathbf{X}^{(1)}\left|\mathbf{X}^{(2)}\right| \cdots \mid \mathbf{X}^{(242)}\right] \in \mathbb{R}^{16 \times 242}$ and the second scientist's observations are stored in the matrix $Y^{(242)}=\left[\mathbf{Y}^{(1)}\left|\mathbf{Y}^{(2)}\right| \cdots \mid \mathbf{Y}^{(242)}\right] \in$ $\mathbb{R}^{16 \times 242}$. (When we replicate our experiment, the identities of the two most correlated animals in each dish don't change from one experiment replication to the next, but which of the two animals is assigned to the first scientist are independent Bernoulli $\left(\frac{1}{2}\right)$ trials.)

For each embedding dimension $k=1,2, \ldots, 16$, we use PCA to generate $\mathcal{A}^{(242)}$ and $\mathcal{B}^{(242)}$, and then we compute $d^{2}\left(\mathcal{A}^{(242)}, \mathcal{B}^{(242)}\right)$ and $\epsilon^{2}\left(\mathcal{X}^{(242)}, \mathcal{Y}^{(242)}\right)$ in the manner described in Section 3.1. Performing 10000 Monte-Carlo replications of this experiment, we plot in Figure 3 the val- 


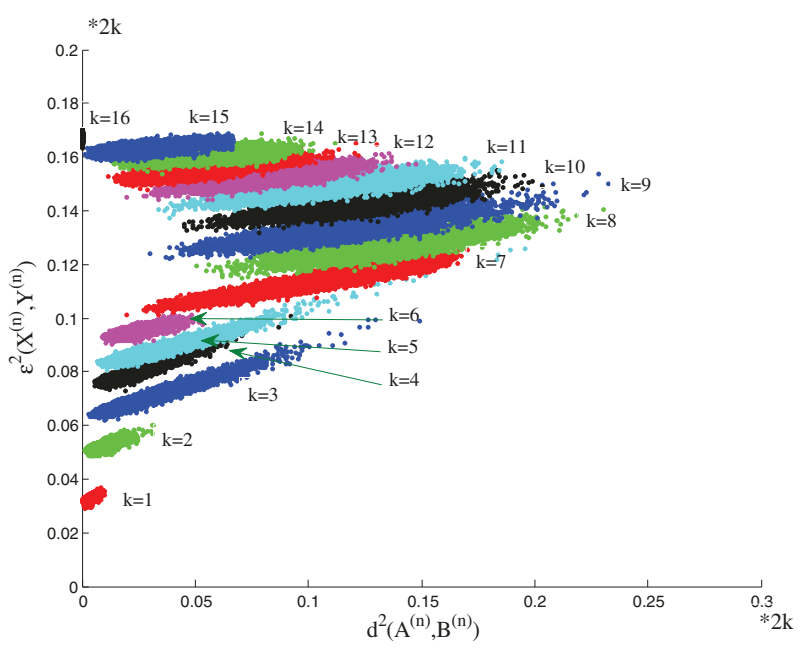

Figure 3. An illustration of the incommensurability phenomenon. Plots of $\epsilon^{2}\left(\mathcal{X}^{(242)}, \mathcal{Y}^{(242)}\right)$ vs $d^{2}\left(\mathcal{A}^{(242)}, \mathcal{B}^{(242)}\right)$ for 10000 Monte-Carlo replicates using the Drosophila larvae data, for each embedding dimension $k=1,2,3, \ldots, 16$. Notice the change from $k=6$ to $k=7$. (Also note that the axis-values are to be multiplied by $2 k$ for the respective values of $k$, since the ranges of $\epsilon^{2}\left(\mathcal{X}^{(242)}, \mathcal{Y}^{(242)}\right)$ and $d^{2}\left(\mathcal{A}^{(242)}, \mathcal{B}^{(242)}\right)$ are the interval $[0,2 k])$.

ues of $\epsilon^{2}\left(\mathcal{X}^{(242)}, \mathcal{Y}^{(242)}\right)$ against the values of $d^{2}\left(\mathcal{A}^{(242)}, \mathcal{B}^{(242)}\right)$ for each of these 10000 replicates, and for each of the embedding dimensions $k=$ $1,2, \ldots, 16$. The colors of the plotted points are red, green, blue, black, cyan, magenta, red, green, blue, black, cyan, magenta, red, green, blue, black according as the embedding dimension is $k=1,2,3, \ldots, 16$. Note that for each embedding dimension $k=1,2, \ldots, 15$, there is a positive linear correlation in the the plotted points of Figure 3. In particular, note the substantial increase in the standard deviation of $d^{2}\left(\mathcal{A}^{(242)}, \mathcal{B}^{(242)}\right)$ as embedding dimension changed from $k=1$ to $k=2$ to $k=3$, and again from $k=6$ to $k=7$. Although the (normalized) values of $\epsilon^{2}\left(\mathcal{X}^{(242)}, \mathcal{Y}^{(242)}\right)$ seem to be anyway increasing as $k$ increases, it also seems that increases in $\epsilon^{2}\left(\mathcal{X}^{(242)}, \mathcal{Y}^{(242)}\right)$ are also explained by the increased values of $d^{2}\left(\mathcal{A}^{(242)}\right.$, $\left.\mathcal{B}^{(242)}\right)$, as these increased values of $d^{2}\left(\mathcal{A}^{(242)}, \mathcal{B}^{(242)}\right)$ occur. This is the incommensurability phenomenon. Although it is not as dramatic as with the simulated data, it is present in this real-data setting.

For an instantiation of one of the scientist's data, the sample covariance matrix had eigenvalues .06284, .01896, .00988, .00748, .00618, $.00473, .00328, .00312, .00291, .00254, .00244, .00228, .00185, .00162$, $.00140, .00128$. Note that there was a precipitous narrowing of eigengap between the 7th eigenvalue and the 8th eigenvalue; this corresponds to the sudden change in behavior in Figure 3 between embedding dimension $k=6$ and embedding dimension $k=7$. 


\section{Summary and Discussion}

When principal components analysis (PCA) is used for the dimension reduction of two random data sets that are highly-correlated with each other, there is a natural hope that that the projected (and normalized) data sets will be commensurate, in the sense that a Procrustes transformation of one to the other will render it close in distance (according to the strength of correlation in the original data). However, sometimes this Procrustean fitting-error is higher than what might be expected, which is the "incommensurability phenomenon." This may occur when the projections are done separately for the two data sets and there is an insufficient gap between covariance eigenvalues as the more-principal principal components are taken and less-principal principal components are discarded, which can lead to nontrivial variance in the resulting PCA projectors. (Indeed, the Cautionary Tale of Two Scientists from Section 2, with spherical covariance structure, creates a perfect storm.)

Our main result is Theorem 2 , which succinctly quantifies the asymptotic effects of (an adaption of) the Hausdorff distance between the PCA projections, in terms of the strength of the correlation between the original data sets, on the Procrustean fitting-error of the projected data. We then illustrate that highly-correlated data, even with a mild gap in covariance eigenvalues, can appreciably exhibit the incommensurability phenomenon; indeed, what we observe from the simulations is very closely aligned with the asymptotic relationship that we proved.

Awareness of these results is important when decisions are made regarding dimension reduction for separate data sets assumed to represent similar phenomena. For example, in distributed settings it may be assumed that highly correlated large data sets can be merged after dimension reduction, thereby allowing for more computationally efficient data transfer. However, our results indicate that this approach can be disastrous, even when the assumption that the separate data sets are highly correlated is valid.

\section{References}

ANDERSON, T.W. (2003), An Introduction To Multivariate Statistical Analysis (3rd ed.), Hoboken NJ: Wiley Series in Probability and Statistics.

BELKIN, M., and NIYOGI, P. (2003), "Laplacian Eigenmaps for Dimensionality Reduction and Data Representation", Neural Computation, 15(6), 1373-1396.

BORG, I., and GROENEN, P. (2005), Modern Multidimensional Scaling: Theory and Applications, New York NY: Springer-Verlag.

CANDES, E., LI, X., MA, Y., and WRIGHT, J. (2009), "Robust Principal Component Analysis", Journal of ACM, 58(1), 1-37.

CHIKUSE, Y. (2003), Statistics on Special Manifolds, Lecture Notes in Statistics, New York NY: Springer. 
GOLDBERG, Y., and RITOV, Y. (2009), "Local Procrustes for Manifold Embedding: A Measure of Embedding Quality and Embedding Algorithms", Machine Learning, 77, $1-25$.

HARDOON, D., SZEDMAK, S., and SHAWE-TAYLOR, J. (2004), "Canonical Correlation Analysis: An Overview with Application to Learning Methods", Neural Computation, 16, 2639-2664.

HORN, R.A., and JOHNSON, C.R. (1990), Matrix Analysis, Cambridge UK: Cambridge University Press.

HOTELLING, H. (1936), "Relations Between Two Sets of Variates", Biometrika, 28 (3/4), $321-377$.

JOLLIFFE, I. (2002), Principal Component Analysis (2nd ed.), New York NY: Springer.

LUO, B., and HANCOCK, E.R. (1999), "Feature Matching with Procrustes Alignment and Graph Editing", Seventh International Conference on Image Processing and Its Applications, 465, 72-76.

O'LEARY, T., and MARDER, E. (2014), "Mapping Neural Activation Onto Behavior in an Entire Animal", Science, 344(6182), 372-373.

PRIEBE, C.E., MARCHETTE, D.J., MA, Z., and ADALI, S. (2013), "Manifold Matching: Joint Optimization of Fidelity and Commensurability", Brazilian Journal of Probability and Statistics, 27(3), 377-400.

QIU, L., ZHANG, Y., and LI, C-K. (2005), "Unitarily Invariant Metrics on the Grassmann Space”, SIAM Journal on Matrix Analysis and Application, 27(2), 507-531.

SAUL, L., and ROWEIS, S. (2000), "Nonlinear Dimensionality Reduction by Locally Linear Embedding", Science, 290, 2323-2326.

SHARMA, A., KUMAR, A., DAUME, H., and JACOBS, D.W. (2012), "Generalized Multiview Analysis: A Discriminative Latent Space", in Proceedings of the IEEE Conference on Computer Vision and Pattern Recognition (2012), pp 2160-2167.

SHEN, C., SUN, M., TANG, M., and PRIEBE, C.E. (2014), "Generalized Canonical Correlation Analysis for Classification", Journal of Multivariate Analysis, 130, 310-322.

SIBSON, R. (1978), "Studies in the Robustness of Multidimensional Scaling: Procrustes Statistics", Journal of the Royal Statistical Society, Series B, 40(2), 234-238.

SIBSON, R. (1979), "Studies in the Robustness of Multidimensional Scaling: Perturbation Analysis of Classical Scaling", Journal of the Royal Statistical Society, Series B, 41(2), 217-229.

SUN, M., PRIEBE, C.E., and TANG, M. (2013), "Generalized Canonical Correlation Analysis for Disparate Data Fusion”, Pattern Recognition Letters, 34(2),194-200.

SUN, M., and PRIEBE, C.E. (2013), "Efficiency Investigation of Manifold Matching for Text Document Classification", Pattern Recognition Letters, 34(11), 1263-1269.

TENENBAUM, J., DE SILVA, V., and LANGFORD, J. (2000), “A Global Geometric Framework for Nonlinear Dimension Reduction", Science, 290, 2319-2323.

VOGELSTEIN, J.T., PARK, Y., OHYAMA, T., KERR, R., TRUMAN, J.W., PRIEBE, C.E., and ZLATIC, M. (2014), "Discovery of Brainwide Neural-Behavioral Maps via Multiscale Unsupervised Structure Learning", Science, 344(6182), 386-392.

WANG, C., LIU, B., VU, B., and MAHADEVAN, S. (2012), "Sparse Manifold Alignment", Technical Report UM-CS-2012-030, University of Massachusetts Department of Computer Science.

WANG, C., and MAHADEVAN, S. (2008), "Manifold Alignment Using Procrustes Analysis", in Proceedings of the 25th International Conference on Machine Learning, pp $1120-1127$. 
WITTEN, D., TIBSHIRANI, R., and HASTIE, T. (2009), “A Penalized Matrix Decomposition, with Applications to Sparse Principal Components and Canonical Correlation Analysis", Biostatistics, 10(3), 515-534.

ZOU, H., and HASTIE, T. (2006), "Sparse Principal Component Analysis", Journal of Computational and Graphical Statistics, 15(2), 262-286. 